
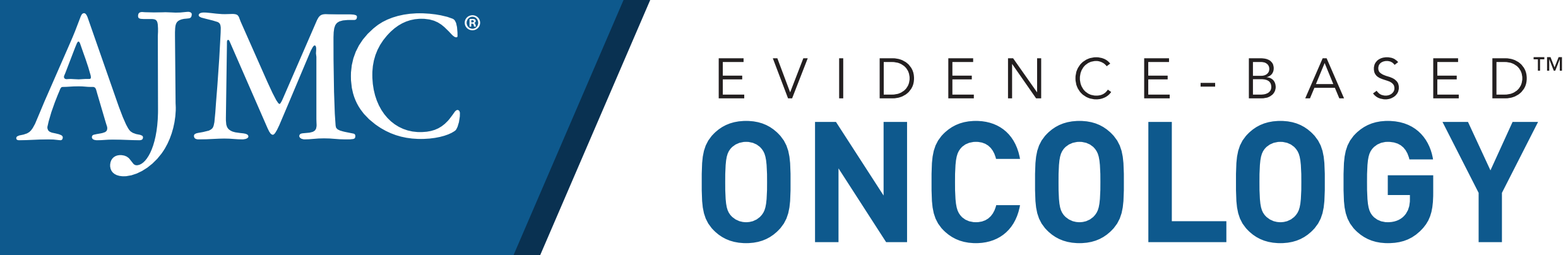

FEBRUARY 2021 VOL. 27 . NO. 2

\title{
ALSO IN THIS ISSUE
}

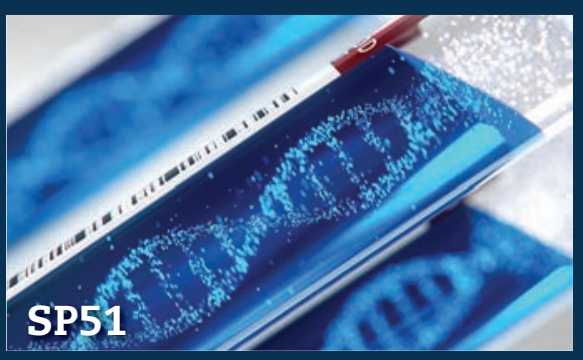

OPPORTUNITIES IN GENE THERAPY. More than 3 years after FDA approved the first chimeric antigen receptor (CAR) T-cell therapy, the number of gene and cell therapies seems poised to soar, with approvals coming in cancer and other conditions, SP51.

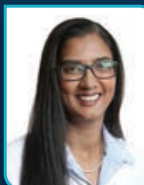

LESS TOXICITY. Tanya Siddiqi MD, of City of Hope, explains how the newly approved CAR T-cell therapy liso-cel promises effectiveness in refractory large B-cell lymphoma with less likelihood of serious adverse events from cytokine release syndrome, SP52.

IMMUNOTHERAPY IN LUNG CANCER. A study shows that certain patients with non-small cell lung cancer are more likely to respond to novel treatments than others, based on the presence of a human leukocyte antigen, SP55. 


\section{Flying the Plane While You Build It}

ASSOCIATE EDITOR

KASHYAP PATEL, MD

President

Carolina Blood and Cancer Care Associates Rock Hill, SC

MICHAELE. CHERNEW, PHD Department of Health Care Policy Harvard Medical School Boston, MA

BRUCE A. FEINBERG, DO

BRUCEA. FEINBERG, DO

Vice President and Chief Medical Office
Cardinal Health Specialty Solutions

Atlanta, GA

A. MARK FENDRICK, MD A. MARK FEN D RICK,
Professor of Medicine and Health Management and Policy Schools of Medicine \& Health University of Michigan Ann Arbor, MI

JOHN FOX, MD, MHA Medical Director for Managed Care OneOncology

Nashville, TN

\section{BO GAMBLE}

Director of Strategic Practice Initiatives

Community Oncology Alliance

Washington, DC

LUCIO GORDAN, MD

Managing Physician and Presiden

Florida Cancer Specialists

Gainesville, FL

JOHN HORNBERGER, MD, MS

Cedar Associates, LLC

Menlo Park, CA

IRA M. KLEIN, MD, MBA

Chief Medical Officer

Health New England

Springfield, Massachusetts

MICHAEL KOLODZIEJ, MD Vice President and Chief Innovation Officer ADVI Health LLC

Washington, DC

ELLEN MATLOFF, MS, CGC President and CEO My Gene Counsel North Haven, CT

JOSHUA J. OFMAN, MD, MSHS Chief of Corporate Strategy and External Affairs GRAIL, Inc

Menlo Park, CA

KATHY OUBRE

Chief Operations Officer
Pontchartrain Cancer Cente

Covington, LA

DEBRA PATT, MD, MPH, MBA

Texas Oncology Cancer Center

Austin, TX

ANDREW L. PECORA, MD, FACP, CPE

Chief Executive Officer

Outcomes Matter Innovations, LLC

Hackensack, NJ

STEPHEN M . SCHLEICHER, MD, MBA

Medical Oncologist

Medical Director for Value-Based Care
OneOncology

OneOncology

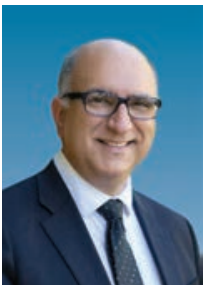

WE ARE IN THE GREATEST PERIOD of innovation cancer care to date. Each day, there is an advance in genomic testing, patient risk stratification, a targeted therapeutic or an immuno-oncologic agent, a cellular therapeutic, or a new approach innovation is far outpacing our ability to systematically assimilate these treatments into practice and align payment in an effective way to incentivize an ever-evolving definition of best practice. As current systems struggle to adapt to this new reality, the culture of medicine and systems of care are, thankfully, inherently conservative. There is a sense of caution around new technologies and payment models that is frequently at odds with the ever-accelerating pace of innovation. The "plan-do-check-act" culture is deeply embedded in our systems of care. Far too often during my residency and fellowship programs, I heard senior attending physicians use the term "bold" as a pejorative indictment rather than a compliment. This desire to ensure patient safety is essential; yet, there are enough patients whose unmet needs might be served by rapid integration of new technologies that we find ourselves at a tension point in the evolution of cancer care.

In Silicon Valley, where former apricot and cherry orchards now burst with silicon wafers and lines of code that have changed human experience, innovators rally around the idea of 'flying the plane while you build it." This paradigm is "rooted in the idea of 'iterative' software development-ship it and fix it and ship it again - in contrast to the earlier approach of tinkering for years and finally shipping." ${ }^{1}$ This model does not ignore safety, functionality, or investor dollars; instead, it is grounded in the need to transcend the limitations of a slower, more plodding model of producing change. The metaphor is an apt one for bringing innovation at a faster pace to cancer care-while we should never undermine safety, our patients' urgent needs demand that we move faster and even embrace a sense of "boldness" in moving forward.

In this issue of Evidence-Based Oncology ${ }^{\mathrm{TM}}$ we see a foreshadowing of what this future could look like and how we may learn to move forward, safely to care. This astounding rate of at an ever-accelerating pace. With this issue of $E B O$, we shift from cover themes to regular departments; both our Targeted Therapies \& Immuno-Oncology section, featuring an interview with Tanya Siddiqi, $\mathrm{MD}$, and our AJMCtv section cover advances in treatment technology that promise more effective, less toxic treatments for patients with unmet needs. In our Precision Medicine section, we review advances in lung cancer treatment, empow ered by innovations in genomic testing, that have improved patient survival and quality of life for patients with advance cancer. Our Payment Model feature reviews the challenges of bringing cost transparency and predictability to cancer care in the area of radiation oncology. In our Pathways interview with the health care quality visionary and former CMS Administrator Don Berwick, MD, MPP, we learn about the importance of using carefully curated clinical data to create a more robust risk-segmentation model to discern the relationship between the individual clinical risk of a patient and the cost of care.

Given the transcendent technologies arriving in the cancer care domain, we need to embrace a new boldness in integrating and incentivizing patient access to care. This demands evolution of our payment systems to recognize the unique risk and clinical needs of patient care, while reducing low value care in our system. It is possible to create a system that brings speed, safety, access, and innovation to patients impacted by a diagnosis of cancer. The ability to navigate the opportunities and challenges of this new paradigm brings with it the ability to improve care disparities, increase the value-centricity of care, and ensure that care becomes iteratively and more deeply patient-centered.

Joseph Alvarnas, $M D$

\section{REFERENCE}

1. Walker R. Build the plane while you're flying it. The Christian Science Monitor. Published March 24, 2016. https://www.csmonitor. com/The-Culture/The-Home-Forum/2016/0324/Build-the-planewhile-you-re-flying-it. Accessed February 4, 2021.

\section{EDITORIAL MISSION}

To present policy makers, payers, and providers with the clinical, pharmacoeconomic, and regulatory information they need to improve efficiency and outcomes in cancer care.

Opinions expressed by authors, contributors, and advertisers are their own and not necessarily those of Clinical Care Targeted Communications, LLC, d/b/a Managed Care \& Healthcare Communications, LLC, the editorial staff, or any member of the editorial advisory board. Clinical Care Targeted Communications, LLC, $\mathrm{d} / \mathrm{b} / \mathrm{a}$ Managed Care \& Healthcare Communications, LLC, is not responsible for accuracy of dosages given in articles printed herein. The appearance of advertisements in this journal is not a warranty, endorsement, or approval of the products or services advertised or of their effectiveness, quality, or safety. Clinical Care Targeted Communications, LLC, d/b/a Managed Care \& Healthcare Communications, $\mathrm{UC}$, dischicts

The content contained in this publication is for general information purposes only. The reader is encouraged to confirm the information presented with other sources. Evidence-Based Oncology $y^{\mathrm{T} M}$ makes no representations or warranties of any kind about the completeness, accuracy, timeliness, reliability, or suitability of any of the information, including content or advertisements, contained in this publication and expressly disclaims liability for any errors and omissions that may be presented in this publication. Evidence-Based Oncology ${ }^{\text {tw }}$ reserves the right to alter or correct any error or omission in the information it provides in this publication, without any obligations. Evidence-Based Oncology'm further disclaims any and al expressed in this pulicirect, consequential, special, exemplary, or other damages arising from the use or misuse of any material or infor 


\section{TABLE OF CONTENTS}

ajmc.com/about/ebo

\section{EVIDENCE-BASED ONCOLOGY \\ FEBRUARY 2021}

Volume 27, Issue 2

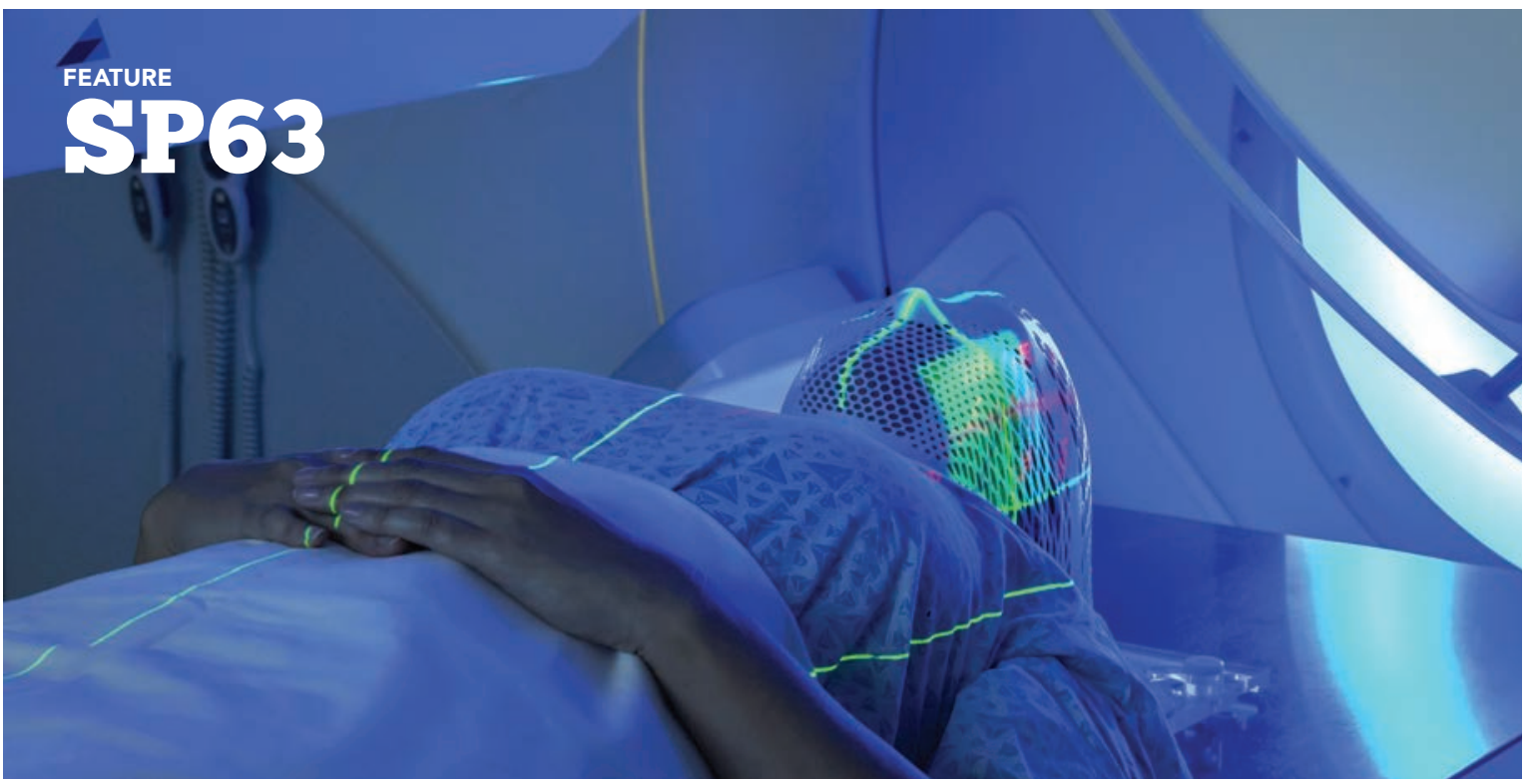

A payment payment model in radiation oncology must be about delivering better care, not just cutting costs, according to a leading professional society.

\section{SP43}

FROM THE EDITOR-IN-CHIEF Flying the Plane While You Build It JOSEPH ALVARNAS, MD

\section{SP45}

FROM THE CHAIRMAN

Our Commitment to Covering Gene Therapies

\section{FEATURES}

\section{SP63}

\section{PAYMENT MODELS}

Radiation Oncology's Role in the Next Chapter of Payment Reform

ANNE HUBBARD, MBA

\section{SP64}

Field Reports: Comments on Oncology Care Model Progress

\section{SP65}

\section{PATHWAYS}

Use of COTA Nodal Address Highlights Variance in Cancer Care Cost: An Interview With Donald M. Berwick, MD, MPP

MARY CAFFREY

\section{SP67}

POLICY

ACCC and ASCO Collaborative

AJMC Targets Disparities in Cancer Clinical Trials

ROSE MCNULTY

\section{INSIDE THE ISSUE}

\section{SP46}

PRACTICE TRANSFORMATION / PEER-REVIEWED

Cost of Home vs Clinic

Administration of Paclitaxel in

\section{Metastatic Breast Cancer}

DOLLY SHARMA, PHD; JEFFREY WOJTYNEK, PHARMD; KATHLEEN M. FOX, PHD; CHELSEA COOPER, PHARMD AND IGONI DOKUBO, MD

\section{SP51-SP54}

TARGETED THERAPY /

IMMUNO-ONOLOGY

The Untapped Potential of Cell and Gene Therapy

LARRY HANOVER

Tanya Siddiqi, MD, Discusses the Promise of Reduced Toxicity With Liso-Cel

MARY CAFFREY AND ROSE MCNULTY

\section{AJMC \\ THE AMERICAN JOURNAL OF MANAGED CARE}

P UB LICATION STAF F
EDITORIAL DIRECTOR

Laura Joszt

ASSOCIATE EDITORIAL DIRECTOR

Mary K. Caffrey

EDITOR

Rose McNulty

ASSOCIATE EDITOR

Maggie L. Shaw

PROJECT MANAGER

Jessica Toye

COPY CHIEF

Jennifer Potash
COPY SUPERVISORS Rachelle Laliberte Paul Silverman

SENIOR COPY EDITOR Kelly King COPY EDITORS

Cheney Baltz

Georgina Carson

Rebekah Harrison Kirsty Mackay Ron Panarotti

CREATIVE DIRECTOR PUBLISHING Melissa Feinen

ART DIRECTOR Julianne Costello

SALES \& MARKETING

VICE PRESIDENT Gilbert Hernandez NATIONAL ACCOUNT MANAGER Ryan O'Leary

OPERATIONS \& FINANCE
NATIONAL ACCOUNT ASSOCIATES Kevin George Carly Mauro

\section{CIRCULATION DIRECTOR Jon Severn}

VICE PRESIDENT, FINANCE Leah Babitz, CPA CONTROLLER Katherine Wyckoff

CORPORATE OFFICERS CHAIRMAN \& FOUNDER Mike Hennessy Sr

VICE CHAIRMAN

Jack Lepping

PRESIDENT \& CEO CHIEF FINANCIAL OFFICER Neil Glasser, CPA/CFE

CHIEF MARKETING OFFICER Michael Baer

EXECUTIVE VICE PRESIDENT, GLOBAL MEDICAL AFFAIRS AND CORPORATE DEVELOPMENT Joe Petroziello

SENIOR VICE PRESIDENT, CONTENT Silas Inman Mike Hennessy Jr

\section{SENIOR VICE PRESIDENT,} Michael Ball

SENIOR VICE PRESIDENT, I.T. \& ENTERPRISE SYSTEMS John Moricone VICE PRESIDENT, HUMAN RESOURCES AND ADMINISTRATION Shari Lundenberg VICE PRESIDENT, MERGERS \& ACQUISITIONS Chris Hennessy EXECUTIVE CREATIVE DIRECTOR, CREATIVE SERVICES Jeff Brown OPERATIONS

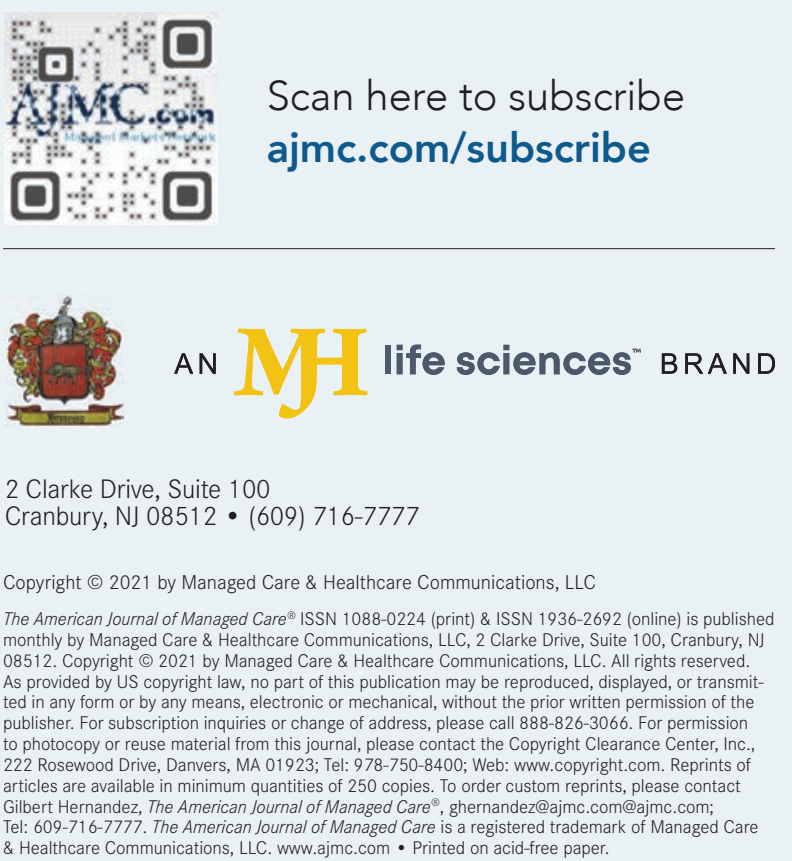




\section{Our Commitment to Covering Gene Therapies}

READERS OF EVIDENCE-BASED ONCOLOGYTM are familiar with regular updates on chimeric antigen receptor (CAR) T-cell therapy and the surrounding challenges in reimbursement. With this issue, we present the commitment of MJH Life Sciences ${ }^{\mathrm{TM}}$ to covering developments in gene therapies beyond their role in cancer care-from sickle cell disease, to cardio fibrosis, to autoimmune conditions, and more.

A little more than 3 years ago, then-FDA Commissioner Scott Gottlieb, MD, heralded a "new frontier" in announcing approval of the first CAR T-cell therapy, tisagenlecleucel, to treat Bcell precursor acute lymphoblastic leukemia. A second therapy, axicabtagene ciloleucel (axi-cel), came shortly after that for diffuse large B-cell lymphoma, but it took until the summer of 2020 for the FDA to approve a third therapy, brexucabtagene autoleucel, for mantle cell lymphoma.

But it appears that the world of gene and cell therapy is ready to explode. The number of treatments and the variety of conditions to be treated are expected to soar in the next few years. This issue includes an overview, "The Untapped Potential of Cell and Gene Therapy," as well as a focused interview with leading scientist Tanya Siddiqi, MD, of City of Hope, our Strategic Alliance Partner in Duarte, California. This National Cancer Institute-designated cancer center has been a pioneer in development of CAR T-cell therapy; Siddiqi was an investigator for ZUMA-1, which led to the approval of axi-cel, and the TRANSCEND NHL trial for lisocabtagene maraleucel (liso-cel), which analysts expect will be approved early this year.

Of course, this is just the beginning. MJH Life Sciences $^{\mathrm{TM}}$ has created GeneTherapyLive.com, a multidisciplinary platform to connect readers with updates, interviews, and other content from across our brands that relate to gene and cell therapies. Among other topics, GeneTherapyLive.com will cover issues in oncology, neurology, ophthalmology, hematology, enzyme disorders, and reimbursement.

Gene and cell therapy are interconnected with precision medicine, and this issue features an interview with Donald M. Berwick, MD, the Harvard Medical School professor and former CMS administrator. Investigators from Cota found wide variation in cancer care delivery, even within the same hospital system, with patients not getting on the right pathway or receiving too much ancillary care. As the use of gene and call therapies increases, cost is an unavoidable roadblock-which makes the need to limit unnecessary variation in care more important than ever. The Cota Nodal Address can offer a good start, Berwick reports.

Enjoy this issue of Evidence-Based Oncology ${ }^{\mathrm{TM}}$, and we welcome your feedback on GeneTherapyLive.com. •

\section{EVIDENCE-BASED ONCOLOGY FEBRUARY 2021}

Volume 27 , Issue 2

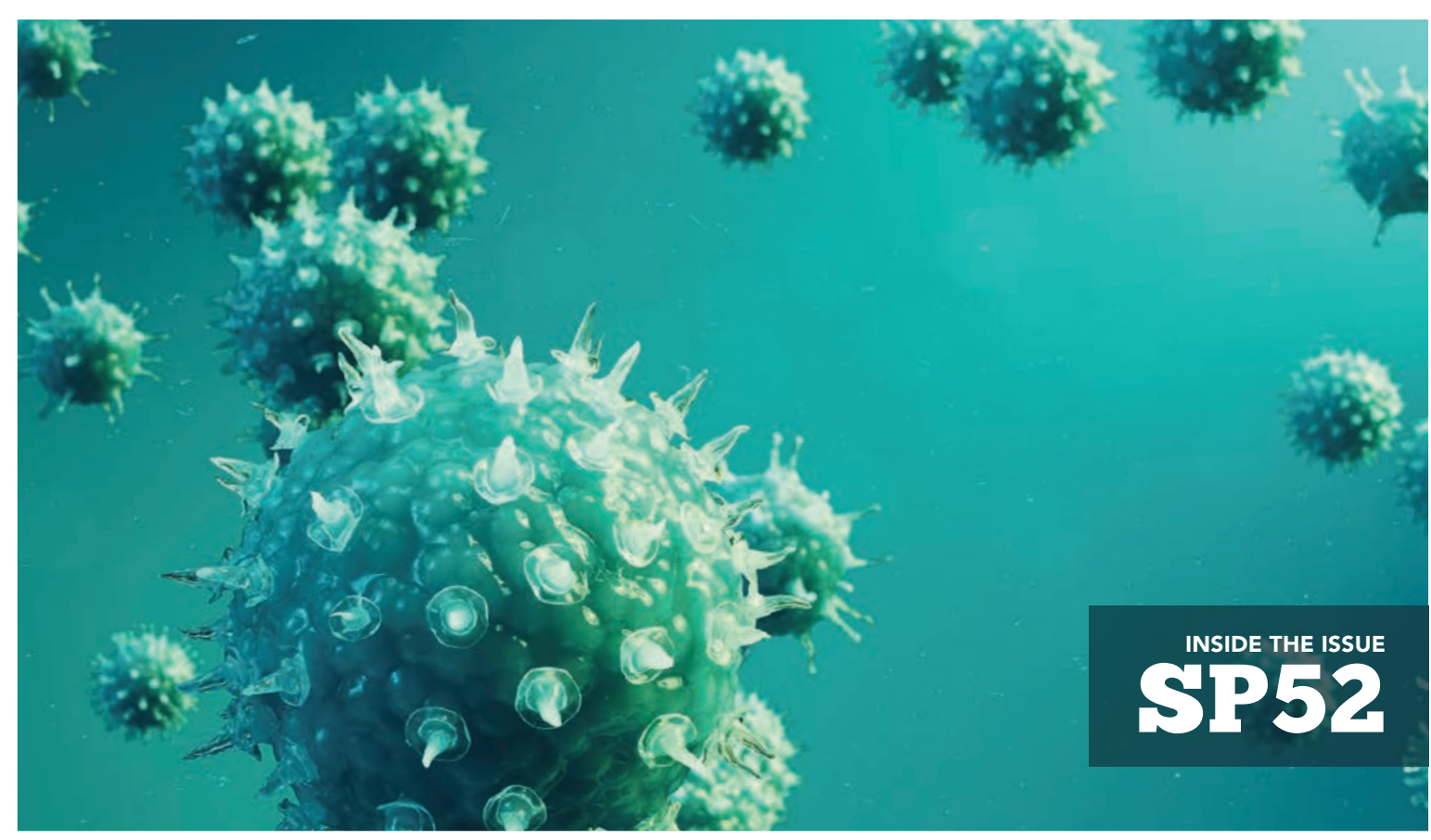

Development of gene and cell therapies is expected to take off in the next few years. Bristol-Myers Squibb received approval for a chimeric antigen receptor (CAR) T-cell therapy for recurrent large B-cell lymphoma that has less toxicity than earlier CAR T-cell products.

CONTINUED FROM SP44

\section{SP55-SP56}

PRECISION MEDICINE

FDA Approves Osimertinib for Adjuvant Treatment of Early-Stage EGFR-Mutated NSCLC

Certain NSCLC Patients Are More Likely to Respond to Immunotherapy, Study Results Show

Possible Treatment Is Identified for Aggressive NSCLC Subset

ROSE MCNULTY

\section{SP57}

\section{FOR EMPLOYERS}

A Practical Path Forward to More Patient-Centered Value Assessment

SACHIN KAMAL-BAHL, PHD, AND BRYAN LUCE, PHD, MBA

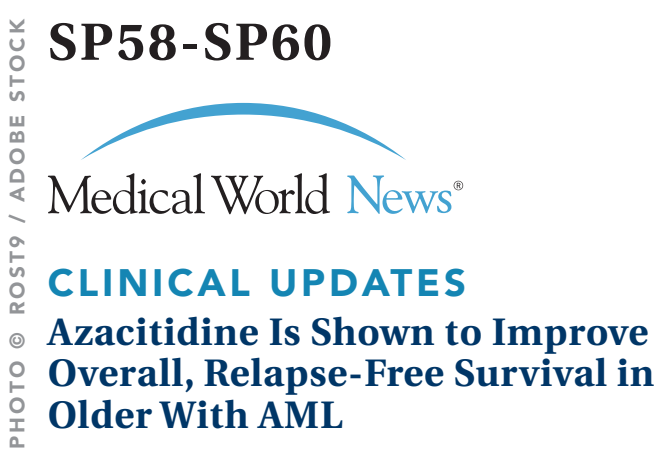

FDA Grants Tiragolumab Breakthrough Therapy Designation for PD-L1 High NSCLC

FDA Updates Ibrutinib Label With 5-Year Data in Waldenström Macroglobulinemia

MANAGED CARE UPDATES As Mammography Screenings Drop, Advanced Breast Cancer Cases Increase in First Months of COVID-19

Lung Cancer Screenings Fall, Biopsy Rates Rise During COVID-19

Proximity to Treatment, Racial Disparities Are Seen in Ovarian Cancer Survival

SP61-SP62

AJMCiv

Bradley Monk, MD, FACOG, FACS on PARP Inhibitors in Ovarian Cancer Treatment

Neeraj Agarwal, MD, Discusses PARP Inhibition in Prostate Cancer 


\title{
Cost of Home vs Clinic Administration of Paclitaxel in Metastatic Breast Cancer
}

\author{
DOLLY SHARMA, PHD; JEFFREY WOJTYNEK, PHARMD; KATHLEEN M. FOX, PHD; \\ CHELSEA COOPER, PHARMD; AND IGONI DOKUBO, MD
}

\section{Introduction}

According to National Comprehensive Cancer Network guidelines, sequential single-agent cytotoxic chemotherapy, such as paclitaxel or protein-bound paclitaxel, is recommended for recurrent or metastatic breast cancer $(\mathrm{mBC}){ }^{1}$ For patients with incurable malignancies, when oral drugs' efficacy and adverse effect (AE) profiles are comparable with those of intravenous (IV) options, the oral drugs are perceived to afford a greater quality of life. ${ }^{2}$ Benefits of oral administration may include patient convenience and reduction of discomfort due to avoidance of hospital and infusion center visits for invasive drug administration. Avoidance of IV drug administration eliminates the risk of infusion reactions and the need for premedication with agents such as steroids and antihistamines., ${ }^{3,4}$ In addition, oral chemotherapy might be associated with cost savings, given that less time is required in clinic because of home administration. ${ }^{5}$ Among IV and oral chemotherapies of similar efficacy, surveys have shown that most patients would prefer oral over IV chemotherapy. ${ }^{2,6}$

To date, oral formulations of agents such as paclitaxel have not been possible because of poor absorption into the bloodstream as a result of the active excretion by P-glycoprotein (P-gp). P-gp is an active efflux pump that prevents the absorption of substrates (such as taxanes) across the intestinal epithelium into the systemic circulation. ${ }^{7}$ Encequidar is an investigational, potent, highly selective P-gp inhibitor that blocks intestinal P-gp, allowing for better absorption of oral paclitaxel into the bloodstream, thereby eliminating the infusion-related AEs associated with IV paclitaxel. ${ }^{8}$ Oral paclitaxel and encequidar is the first oral taxane plus P-gp inhibitor that demonstrated a significant improvement in confirmed overall response rate and overall survival compared with IV paclitaxel in findings from its phase 3 trial. Compared with IV paclitaxel, this oral therapy was also associated with a lower risk of neuropathy and alopecia but a higher incidence of hematologic and gastrointestinal AEs. ${ }^{8}$

The objective of this study was to estimate the costs associated with home administration of oral paclitaxel and encequidar compared with clinic/office administration of IV paclitaxel $\left(175 \mathrm{mg} / \mathrm{m}^{2}\right)$ and protein-bound paclitaxel from a US payer perspective in patients with $\mathrm{mBC}$.

\section{Methods}

\section{Model Structure and Assumptions}

A Microsoft Excel-based cost-calculator model was constructed from a US payer perspective to calculate aggregated costs associated with home administration of oral therapy vs clinic/office administration of IV therapy over a 6-month treatment duration (Figure 1).

The target population for this model included patients with $\mathrm{mBC}$ with any receptor status. For the base case analysis, a health plan with 10,000 treated patients with $\mathrm{mBC}$ was assumed, with $30 \%$ assumed to have Medicare insurance. The model evaluates patients starting on the following treatments:

- (A) ORAL PACLITAXEL AND ENCEOUIDAR: home administration (both medications are taken once daily for 3 consecutive days each week)

- (B) IV PACLITAXEL (175 MG/M²): office/clinic infusion (once every 3 weeks)

- (C) PROTEIN-BOUND PACLITAXEL (260 MG/M²): office/clinic infusion (once every 3 weeks)

The cost-calculator model included all direct costs (pertaining to payers) related to administration of the oral or IV therapies; these included drug administration (drug preparation and equipment, supplies and resources required for administration), premedications and concomitant medications, oncologist office visits, laboratory testing, and administration-related AEs. Administrationrelated costs included any costs associated with the administration, including use of local anesthesia; IV access; access to indwelling IV, subcutaneous catheter or port; flush at conclusion of infusion; standard tubing, syringes, and supplies; and preparation of chemotherapy agent. ${ }^{9}$ Laboratory testing included routine

\begin{abstract}
OBJECTIVES: To estimate the costs associated with home administration of oral paclitaxel and encequidar (novel P-glycoprotein pump inhibitor allowing oral paclitaxel bioavailability) compared with clinic/office administration of intravenous (IV) paclitaxel $\left(175 \mathrm{mg} / \mathrm{m}^{2}\right)$ and protein-bound paclitaxel in US patients with metastatic breast cancer.
\end{abstract}

\section{STUDY DESIGN: Economic analysis.}

METHODS: A cost calculator was constructed from a payer's perspective including all costs related to administration of the chemotherapies, including drug administration, premedications and concomitant medications, oncologist office visits, laboratory testing, and administration-related adverse events. Total administration cost per patient per month (PPPM) and 6-month costs per patient were estimated for oral paclitaxel and encequidar, $175 \mathrm{mg} / \mathrm{m}^{2}$ IV paclitaxel, and protein-bound paclitaxel. Three scenarios for oral paclitaxel and encequidar, a weekly IV paclitaxel scenario $\left(80-100 \mathrm{mg} / \mathrm{m}^{2}\right)$, and univariate sensitivity analyses were conducted.

RESULTS: Home administration of oral paclitaxel and encequidar was associated with a total administration cost of $\$ 523$ PPPM $64.4 \%$ lower than once-every-3-weeks IV paclitaxel $\left(175 \mathrm{mg} / \mathrm{m}^{2}\right.$; $\$ 1469$ PPPM) and 63.8\% lower than protein-bound paclitaxel (260 mg/m²; \$1445 PPPM). Difference in costs was driven largely by higher administration and premedication costs associated with IV therapies. Scenario analyses showed that increased clinical experience with home administration of oral paclitaxel and encequidar was associated with reduction in cost of care associated with its administration over time. For the weekly IV $\left(80-100 \mathrm{mg} / \mathrm{m}^{2}\right)$ paclitaxel scenario, the total administration cost was $\$ 2510$ PPPM (4.8 times higher than for oral paclitaxel and encequidar). Univariate sensitivity analysis demonstrated that the model findings were robust.

CONCLUSIONS: Home administration of oral paclitaxel and encequidar was associated with lower administration costs compared with once-every-3-weeks IV paclitaxel $\left(175 \mathrm{mg} / \mathrm{m}^{2}\right)$ and proteinbound paclitaxel, resulting in potential cost savings for payers. 
tests conducted alongside chemotherapy, including complete blood count (CBC) and comprehensive metabolic panel (CMP). Only AEs of grade 3 or 4 directly related to administration route (oral and IV) were included in the model (ie, allergic and injection site/infusion reactions, nausea/vomiting, diarrhea).

\section{Assumptions}

The model considers only outcomes and costs that are associated with the specific route and setting of administration of each drug therapy, including severe administration-related AEs (ie, grade 3 or 4). Since the focus of the study was to estimate costs associated with administration only, the drug costs of encequidar, oral paclitaxel, IV paclitaxel, and protein-bound paclitaxel were not included in the analysis. The drug-specific clinical efficacy (eg, overall survival) and AEs (other than administration-related) were considered equal and were not included in this analysis. All patients were assumed to be on chemotherapy for the entire 6 months with $100 \%$ adherence to medication and office visit and lab testing schedules. To make comparisons uniform, each month was assumed to be composed of 28 days, and all therapies were assumed to have started on week 1 of the first month.

\section{Data Sources and Input Parameters}

Table 1 summarizes the input parameters and data sources included in the model. The model was populated with data from 3 primary sources: a targeted review of the peer-reviewed literature, the preliminary $\mathrm{AE}$ rates of oral paclitaxel and encequidar from the phase 3 clinical trial, ${ }^{8}$ and expert oncologist opinion.

Three breast cancer oncologists (experts) based in large academic institutions in different US geographic locations, with a mean of 8 to 10 years of clinical practice experience, were interviewed to obtain their opinions on practice patterns with IV and oral therapies in patients with mBC. Specifically, the interviews aimed to capture data about: (1) drug administration schedule, including premedications; (2) the frequency of oncologist visits, home nurse visits, and lab testing; (3) premedications; (4) monitoring of patients for AEs; and (5) administrationrelated AE risk. Two rounds of teleconference panel sessions were conducted with the experts to obtain data. The first session with each expert was a 1:1 interview, and the second interview session was a group interview that involved discussion of previous answers to reach consensus among the experts.

\section{RESOURCE UTILIZATION}

For oral paclitaxel and encequidar, base case analysis data for frequency of oncologist office visits and lab testing were obtained from expert interviews. The dosing schedule for oral paclitaxel and encequidar was provided by Athenex's product information. For IV paclitaxel $\left(175 \mathrm{mg} / \mathrm{m}^{2}\right)$ and protein-bound paclitaxel, frequency of IV administration was captured from their package inserts. ${ }^{10,11}$ Frequency of oncologist visits and lab testing for IV therapies was derived from expert opinion.

costs

For all therapies, cost of an oncologist visit was derived from the 2020 CMS Physician Fee
Schedule. ${ }^{12}$ Medicare costs for lab testing (CBC and CMP) were derived from the 2020 Clinical Laboratory Fee Schedule. Commercial/ private insurer costs for lab testing were based on mean commercial reimbursement amounts obtained from laboratory pricing and reimbursement data available from Sparrow Health System. ${ }^{33,14}$

Cost for premedication (the antiemetic ondansetron) for oral paclitaxel and encequidar was estimated from 2020 Average Sales Pricing data from CMS. ${ }^{15}$ For IV and proteinbound paclitaxel, administration and premedication/concomitant medication costs were estimated from a retrospective database study evaluating the cost components

for administering a single-agent IV breast cancer drug to women with $\mathrm{mBC}$ in the United States. ${ }^{16}$ Based on the distribution of individual components in mean cost per IV-administration visit, costs for administration and premedication/ concomitant medications were estimated for IV paclitaxel and protein-bound paclitaxel. Premedications/ concomitant medications for IV therapies included antiemetic agents (eg, palonosetron, granisetron), saline solution, dextrose water, corticosteroids (eg, dexamethasone), heparin, antihistamine (eg, diphenhydramine), and histamine-2 receptor antagonists (eg, ranitidine)

Only those AEs of grade 3 or 4 that were related to the specific route of administration were included in the model (ie, allergic and injection site/infusion reactions, nausea/vomiting, diarrhea). The rates of grade 3 or 4 AEs were based on preliminary phase 3 clinical trial data for oral paclitaxel and encequidar, ${ }^{8}$ package inserts for IV and protein-bound paclitaxel, and expert opinion..$^{10,11}$ Administration-related AE costs per event were derived from an oncology budget impact study. This study estimated costs of managing AEs (grade 3 or 4 ) by identifying each $\mathrm{AE}$ with specific diagnosis codes and associated resource use and costs from the Healthcare Cost and Utilization Project data. ${ }^{17}$

\section{Outcomes and Analysis}

Outcomes were assessed over a 6-month treatment duration. The primary outcomes of the model were the total costs associated with home administration of oral paclitaxel and encequidar and clinic/office administration of IV paclitaxel $\left(175 \mathrm{mg} / \mathrm{m}^{2}\right)$ and protein-bound paclitaxel per patient per month (PPPM), as well as 6-month costs per patient. The outcomes were calculated for the entire duration of treatment by summing all individual component costs. All costs were inflated to 2019 US dollars using the Medical Care Consumer Price Index. ${ }^{18}$

In addition, 3 scenario analyses were conducted for oral paclitaxel and encequidar to evaluate the " 
TABLE 1. Model Inputs and Their Data Sources for the Base Case Analysis (6-month administration per patient) of Home Oral Administration vs Clinic/Office IV Administration for mBC

\begin{tabular}{|c|c|c|c|c|}
\hline Variable & $\begin{array}{l}\text { Oral paclitaxel } \\
\text { and encequidar }\end{array}$ & $\begin{array}{l}\text { IV paclitaxel } \\
\left(175 \mathrm{mg} / \mathrm{m}^{2}\right)\end{array}$ & $\begin{array}{l}\text { Protein-bound } \\
\text { IV paclitaxel }\end{array}$ & Source \\
\hline \multicolumn{5}{|l|}{ 6-month resource utilization } \\
\hline Oncologist office visits ( $n$ ) & 9 & 9 & 9 & \multirow{4}{*}{ Expert opinion } \\
\hline \multicolumn{4}{|l|}{ Lab evaluations (n) } & \\
\hline $\mathrm{CBC}$ & 24 & 9 & 9 & \\
\hline CMP & 12 & 9 & 9 & \\
\hline Administration costs & NA & $\$ 522.40$ & $\$ 534.71$ & {$[16]$} \\
\hline \multicolumn{5}{|c|}{ Visits, labs, and premedications: costs } \\
\hline Oncologist office visit & $\$ 151.09$ & $\$ 151.09$ & $\$ 151.09$ & \multirow{7}{*}{ [12-16] } \\
\hline \multicolumn{5}{|l|}{ Lab evaluation (CBC) } \\
\hline Medicare & $\$ 10.60$ & $\$ 10.60$ & $\$ 10.60$ & \\
\hline Private & $\$ 21.28$ & $\$ 21.28$ & $\$ 21.28$ & \\
\hline \multicolumn{4}{|l|}{ Lab evaluation (CMP) } & \\
\hline Medicare & $\$ 14.20$ & $\$ 14.20$ & $\$ 14.20$ & \\
\hline Private & $\$ 40.16$ & $\$ 40.16$ & $\$ 40.16$ & \\
\hline Premedications & $\$ 29.95$ & $\$ 231.78$ & $\$ 176.34$ & \\
\hline \multicolumn{5}{|c|}{ Administration-related AEs (grade 3 or 4 ) } \\
\hline \multicolumn{4}{|c|}{ Allergic and injection site/infusion reaction } & \multirow{9}{*}{$\begin{array}{c}{[7,10,11,17]} \\
\text { expert opinion }\end{array}$} \\
\hline Incidence & NA & $1.0 \%$ & $1.0 \%$ & \\
\hline Cost of managing/event & NA & $\$ 8268.96$ & $\$ 8268.96$ & \\
\hline \multicolumn{4}{|l|}{ Nausea/vomiting } & \\
\hline Incidence & $6.8 \%$ & $0.7 \%$ & $4.0 \%$ & \\
\hline Cost of managing/event & $\$ 7280.00$ & $\$ 7280.00$ & $\$ 7280.00$ & \\
\hline \multicolumn{4}{|l|}{ Diarrhea } & \\
\hline Incidence & $5.3 \%$ & $1.0 \%$ & $1.0 \%$ & \\
\hline Cost of managing/event & $\$ 8167.04$ & $\$ 8167.04$ & $\$ 8167.04$ & \\
\hline
\end{tabular}

$\mathrm{AE}$, adverse event; $\mathrm{CBC}$, complete blood count; $\mathrm{CMP}$, complete metabolic panel; IV, intravenous; $\mathrm{mBC}$, metastatic breast cancer; $\mathrm{NA}$, not applicable.

TABLE 2. Base Case Analysis Results: Home Oral Administration vs Office/Clinic IV Administration for mBC

\begin{tabular}{lccc} 
Outcome & $\begin{array}{c}\text { Oral paclitaxel } \\
\text { and encequidar }\end{array}$ & $\begin{array}{c}\text { IV paclitaxel } \\
\left(\mathbf{1 7 5} \mathbf{~ m g} / \mathrm{m}^{2}\right)\end{array}$ & $\begin{array}{c}\text { Protein-bound } \\
\text { paclitaxel }\end{array}$ \\
\hline Total administration cost: PPPM & $\$ 523$ & $\$ 1469$ & $\$ 1445$ \\
\hline Total 6-month administration cost: per patient & $\$ 3140$ & $\$ 8817$ & $\$ 8669$ \\
(a) Administration costs & $\mathrm{NA}$ & $\$ 4702$ & $\$ 4812$ \\
(b) Visits, labs, and premedications & $\$ 2212$ & $\$ 3900$ & $\$ 3401$ \\
(c) Administration-related AEs & $\$ 928$ & $\$ 215$ & $\$ 456$ \\
\hline Total 6-month administration cost: all patients & $\$ 31,399,411$ & $\$ 88,167,676$ & $\$ 86,688,628$ \\
\hline
\end{tabular}

$\mathrm{AE}$, adverse event; $\mathrm{mBC}$, metastatic breast cancer; NA, not applicable; PPPM, per patient per month.

${ }^{a}$ Total 6-month administration costs per patient are composed of (a) administration costs; (b) visits, labs, and premedications; and (c) administration-related AEs.

impact of varying key clinical inputs on the cost of administration. These scenarios modeled varying frequencies of oncologist office visits and lab testing to demonstrate the impact on cost of home administration once oncologists gained experience with the new regimen. They were (1) decreased frequency of lab testing after 3 months of treatment initiation, (2) decreased frequency of lab testing after 1 month of treatment initiation, and (3) decreased frequency of oncologist visits and lab testing after 1 month of treatment initiation.
In this cost analysis, IV paclitaxel $\left(175 \mathrm{mg} / \mathrm{m}^{2}\right)$, the FDA-approved dose of IV paclitaxel for the treatment of $\mathrm{mBC}$ that is given every 3 weeks, was included to maintain consistency with the phase 3 trial of oral paclitaxel and encequidar. However, an additional "weekly IV paclitaxel" scenario was conducted in which weekly IV paclitaxel $\left(80-100 \mathrm{mg} / \mathrm{m}^{2}\right)$ was included as an alternative regimen because it is another dosage of IV paclitaxel commonly used in clinical practice for the treatment of $\mathrm{mBC}$.
Univariate sensitivity analyses were conducted to determine the extent to which uncertainty inherent to the model inputs affected the modeled outcomes when the inputs were varied 1 at a time. The high and low values of clinical inputs for oral paclitaxel and encequidar (such as oncologist visits and lab testing frequencies) were based on clinical relevance determined by experts or literature. Clinical meaningfulness pertains to minimum or maximum number of oncologist/lab visits that would be required based on clinical experience, so rather than varying these variables by $30 \%$, clinically relevant values were selected.

All other inputs were varied to $30 \%$ higher and $30 \%$ lower than the base case values to analyze the impact on results. Oncologist visits and lab testing frequencies of IV therapies were not varied in the sensitivity analyses, as schedules for those dosing regimens are well established.

\section{Results}

Base Case

The results of the base case analysis are shown in Table 2. The base case analyzed the per-patient per-month (PPPM) cost of home administration of oral paclitaxel and encequidar compared with clinic/office administration of IV paclitaxel (175 mg/ $\mathrm{m}^{2}$ ) and protein-bound paclitaxel for a treatment duration of 6 months for 10,000 patients with mBC with $30 \%$ of patients covered by Medicare.

The total administration costs for oral paclitaxel and encequidar, IV paclitaxel $\left(175 \mathrm{mg} / \mathrm{m}^{2}\right)$, and protein-bound paclitaxel were $\$ 523$ PPPM, $\$ 1469$ PPPM (2.8 times higher than oral paclitaxel and encequidar), and \$1445 PPPM (2.8 times higher than oral paclitaxel and encequidar), respectively. The difference in costs was driven largely by higher costs for administration and for premedication associated with IV therapies. The total administration cost PPPM for oral paclitaxel and encequidar was $64.4 \%$ lower than for IV paclitaxel $\left(175 \mathrm{mg} / \mathrm{m}^{2}\right)$ and $63.8 \%$ lower than for protein-bound paclitaxel.

\section{Scenario Analysis}

The results of the scenario analyses are shown in Table 3. The 3 scenario analyses showed that with decreasing frequency of oncology office visits and lab tests, as oncologists gained more experience with the home administration of oral paclitaxel and encequidar, $7 \%$ to $28 \%$ reductions of the cost of care associated with its administration would occur in the base case analysis. For the weekly IV paclitaxel scenario, the total administration cost for weekly IV paclitaxel $\left(80-100 \mathrm{mg} / \mathrm{m}^{2}\right)$ was $\$ 2510$ PPPM (4.8 times higher than oral paclitaxel and encequidar).

\section{Sensitivity Analysis}

Detailed results of the 1-way sensitivity analysis are shown in Supplementary Table 1. Figure 2

shows the input variables that resulted in maximum variability in home administration of oral paclitaxel and encequidar cost PPPM. Cost (PPPM) of home administration of oral paclitaxel and encequidar varied the most when the frequency and cost of oncologist office visits, frequency of $\mathrm{CBC}$ lab visits, and incidence and cost of nausea/vomiting were 
increased or decreased by approximately $30 \%$. By varying each model input 1 at a time, the cost of home administration of oral paclitaxel and encequidar increased by a maximum of $\$ 68 \mathrm{PPPM}$ compared with the base case analysis, indicating that the model was robust.

\section{Discussion}

In oncology, including mBC, cytotoxic chemotherapy is primarily administered IV rather than orally. ${ }^{19}$ The advantages of oral chemotherapy could be multifold. Compared with IV chemotherapy, it provides ease of administration with respect to timing and location (ie, home setting). Oral chemotherapy also has the potential to provide relatively prolonged treatment exposure as compared with IV infusions. Use of oral chemotherapy can potentially result in reduced health care resource utilization of outpatient infusion-related services. Overall, oral therapy could result in better quality of life than parenteral administration..$^{20}$ However, oral administration of many anticancer agents, including paclitaxel, has not been possible due to poor absorption into the bloodstream due to the active excretion by P-gp in the intestinal cells. ${ }^{7}$ Studies have shown that when administered with encequidar, a highly specific intestinal P-gp inhibitor, oral paclitaxel achieves therapeutic blood levels and is more efficacious than IV paclitaxel in patients with $\mathrm{mBC}$. ${ }^{4,8}$

In the current study, a cost calculator model demonstrated that, from a US payer perspective, home administration of oral paclitaxel and encequidar was associated with much lower total administration cost PPPM than clinic/office administration of IV paclitaxel $\left(175 \mathrm{mg} / \mathrm{m}^{2}\right)$ and proteinbound paclitaxel, assuming equal efficacy and safety other than administration-related AEs. Findings show that home administration of oral paclitaxel and encequidar may result in potential cost savings for payers. The immediate cost savings associated with oral paclitaxel and encequidar from the payer's perspective for a 6 -month treatment duration were approximately $\$ 940$ PPPM compared with IV chemotherapies. The key drivers of cost savings were found to be the lower administration costs and the much lower premedication costs associated with home administration of oral therapy.

The findings from this study are consistent with those of other pharmacoeconomic studies that compared costs associated with oral vs IV chemotherapies. Cassidy et al conducted a cost-effectiveness study of oral capecitabine and IV 5 -fluorouracil/leucovorin (5-FU/LV) in patients with Dukes' C colon cancer, wherein incremental direct costs (ie, chemotherapy, treatment administration visits, hospitalization, and management of AEs) and societal costs (ie, time and travel) were assessed. ${ }^{21}$ The study results showed that compared with 5-FU/ $\mathrm{LV}$, capecitabine was the dominant treatment: It was associated with almost half of the direct costs (£3176 vs $£ 6829$ ), one-fifth of the societal costs (£381 vs £1699), and better outcomes. Although capecitabine had higher acquisition costs, its associated costs for administration, management of AEs, and patient travel and time were much lower than those of 5 -FU/LV. ${ }^{21}$ Similarly, Lay et al conducted a cost minimization analysis (assuming equal efficacy) of oral

TABLE 3. Scenario Analysis for mBC: Home Oral vs Clinic/Office IV Administration

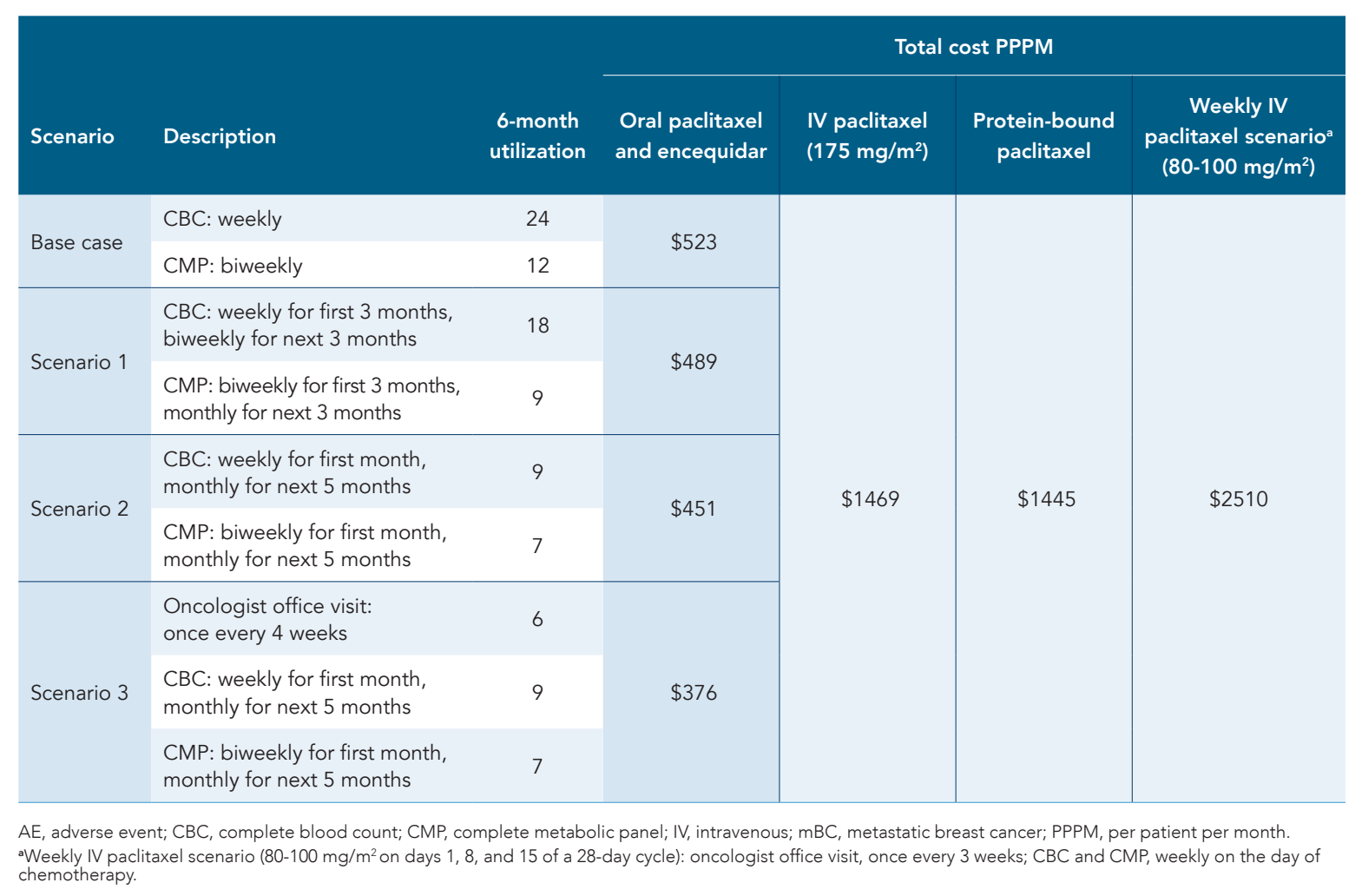

FIGURE 2. Cost PPPM Results of 1-Way Sensitivity Analysis ( $\pm 30 \%)$ : Home Administration of Oral Paclitaxel and Encequidar for $\mathrm{mBC}$

Oncologist office visits

Cost: oncologist office visit

Lab-visit CBC

Incidence-nausea/vomiting

Cost: nausea/vomiting

448

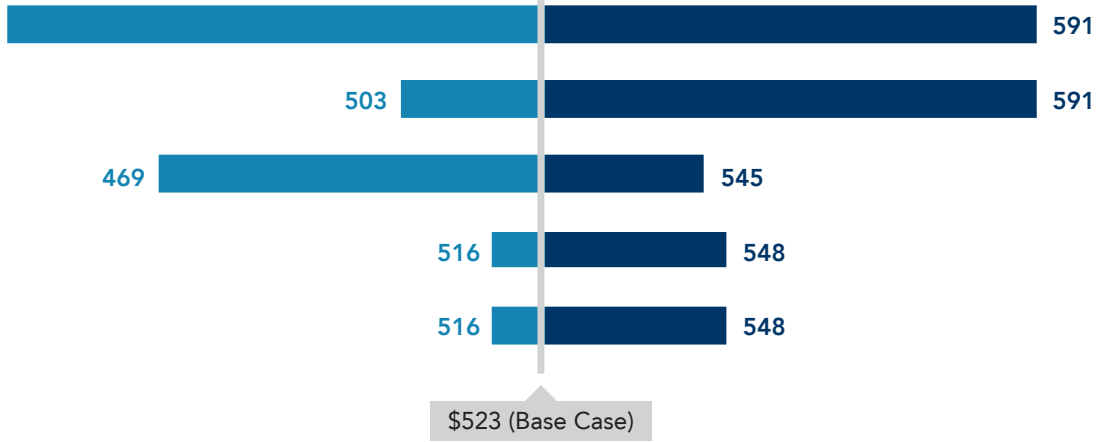

$\mathrm{CBC}$, complete blood count; $\mathrm{mBC}$, metastatic breast cancer; PPPM, per patient per month.

vinorelbine and IV chemotherapies (vinorelbine, gemcitabine, paclitaxel, and docetaxel) for treatment of patients with non-small cell lung cancer. They found that IV chemotherapies were associated with costs per patient that were 1.2 to 2.3 times higher than those of oral vinorelbine (£3746, £5332, £5977, and £6766, respectively, vs £2888). ${ }^{22}$

\section{Strengths and Limitations}

Our study has numerous strengths. The model accounted for all costs related to administration of the treatments, including administration supplies and IV preparation, premedications/concomitant medications, oncologist office visits, laboratory testing, and administration-related AEs. Although real-world practice patterns for our investigational oral drug combination are not yet known, 2 rounds of thorough expert interviews were conducted with oncologists specializing in the treatment of $\mathrm{mBC}$ to understand the potential resource utilization and practice patterns that would be associated with this therapy. Furthermore, in-depth scenario analyses projected to be associated with increased clinical experience of prescribing this new regimen, involving variations in the frequency of oncologist and lab-testing visits, were created; univariate sensitivity analysis was also conducted to test the robustness of the model. In addition to these scenario analyses, an additional comparatorweekly IV paclitaxel $\left(80-100 \mathrm{mg} / \mathrm{m}^{2}\right)$ —was included, as it is a routinely used dose of IV paclitaxel in clinical practice. Findings from all scenario analyses showed cost savings associated with home administration of oral paclitaxel and encequidar.

Since this was an early-stage study aiming to understand costs associated only with administration of oral and IV chemotherapy in different settings, the analysis assumed equal efficacy and safety (other than administration-related AEs) among the therapies, and drug acquisition costs were not included. Differential efficacy and safety profile and inclusion of drug acquisition costs will impact the overall economic value associated with this oral regimen. Once the pricing of oral " 
paclitaxel and encequidar has been set, additional key information for payers will be available from other economic evaluations, including budget impact analysis.

The model was constructed from the perspective of US payers; hence, only payer-related direct costs for each administration setting were included. It will be important to investigate the impact of oral paclitaxel and encequidar from a patient's perspective. These could include both direct costs, such as out-of-pocket expenses for the chemotherapy and travel for infusions, and indirect costs, such as lost productivity associated with IV chemotherapy.

Our study had several limitations. First, only a 6-month treatment duration was assumed in the analysis given the lack of real-world data on treatment duration of oral therapy. It is possible in the real-world setting that the duration of oral chemotherapy could be longer than that of fixed-duration IV chemotherapies. Second, an assumption of $100 \%$ adherence to medication and lab testing was made. While adherence might vary in real-world practices, it was important to make this assumption in the study to understand the complete resource utilization (ie, lab testing, office visits) and costs associated with administration of agents in different settings. Third, cost inputs were derived from multiple sources. However, extensive univariate analyses were done to verify the robustness of the model. Fourth, for the private insurer costs of lab testing, mean commercial reimbursement amounts were used in the analysis. However, there could be a high variability in commercial lab testing costs. Finally, while there may be provider incentives associated with the prescribing of IV therapies, those were not included in the analysis due to high variability.

\section{Conclusions}

This study found that home administration of oral paclitaxel and encequidar was associated with total administration cost of $\$ 523$ PPPM, a cost $64.4 \%$ lower than that of once-every-3-weeks IV paclitaxel $\left(175 \mathrm{mg} / \mathrm{m}^{2}, \$ 1469\right.$ PPPM) and $63.8 \%$ lower than that of protein-bound paclitaxel $\left(260 \mathrm{mg} / \mathrm{m}^{2}\right.$; $\$ 1445$ PPPM). The findings indicate that home administration of oral paclitaxel and encequidar may result in potential cost savings for payers. •
AUTHOR AFFILIATIONS: EPI-Q, Inc (DS), Oak Brook, LL; Athenex, Inc ( AW, ID), Schaumberg II: Mirador G), Oal (KMF, CC), Kennett Square, PA.

AUTHOR DISCLOSURES: Dolly Sharma is an employee of EPI-Q Inc, which received payment from Athenex associated with the development and execution of this study. Jeffrey Wojtynek and Igoni Dokubo are employed by and hold stock in Athenex, which funded the study. Kathleen M. Fox and Chelsea Cooper are employees of Mirado Global, which received payment associated with the execution of this study.

FUNDING SOURCE: This study was sponsored by Athenex, Inc. AUTHOR INFORMATION: Concept and design (DS, JW, $\mathrm{KMF}, \mathrm{CC}, \mathrm{ID})$; acquisition of data (DS); analysis and interpretation of data (DS, JW, KMF, CC, ID): drafting of manuscript (DS); critical revision of manuscript for important intellectual content (DS, JW, KMF, CC, ID) obtaining funding (JW, KMF, ID); administrative, technical, and logistical support (DS); and supervision (JW, KMF, CC, ID).

CORRESPONDING AUTHOR: Dolly Sharma, PhD (dolly. sharma@epi-q.com).

\section{REFERENCES}

1. Pagani O, Senkus E, Wood W, et al; ESO-MBC Task Force. International guidelines for management of metastatic breast cancer: can metastatic breast cancer be cured? J Natl Cancer Inst. 2010;102(7):456-463. doi:10.1093/jnci/djq029

2. Liu G, Franssen E, Fitch MI, Warner E. Patient preferences for oral versus intravenous palliative chemotherapy. J Clin Oncol. 1997;15(1):110-115. doi:10.1200/JCO.1997.15.1.110

3. Eek D, Krohe M, Mazar I, et al. Patient-reported preferences for oral versus intravenous administration for the treatment of cancer: a review of the literature. Patient Prefer Adherence. 2016;10:1609-1621. doi:10.2147/PPA.S106629

4. Jackson CGCA, Deva S, Bayston K, et. al. An international randomized cross-over bio-equivalence study of oral paclitaxel + HM30181 compared with weekly intravenous (IV) paclitaxel in patients with advanced solid tumors. Ann Oncol. 2019;30(suppl 5):v159-v193. doi:10.1093/ annonc/mdz244

5. Cardoso F, Colleoni M, Di Leo A, et al. Oral chemotherapy in advanced breast cancer: expert perspectives on its role in clinical practice. Cancer Treatm Commun. 2016;6(suppl 1):S1-S10. doi:10.1016/S22130896(16)06001-1

6. Schott S, Schneeweiss A, Reinhardt J, et al. Acceptance of oral chemotherapy in breast cancer patients - a survey study. BMC Cancer. 2011:11:129. doi:10.1186/1471-2407-11-129

7. Dai M-S, Chao T-C, Chiu C-F, et al. Oral paclitaxel in the treatment of metastatic breast cancer (MBC) patients. J Clin Oncol. 2019;37(15 suppl):1084. doi:10.1200/JCO.2019.37.15_suppl.1084

8. Umanzor G, Cutler DL, Barrios FJ, et al. Oral paclitaxel with encequidar: the first orally administered paclitaxel shown to be superior to IV paclitaxel on confirmed response and survival with less neuropathy: a phase III clinical study in metastatic breast cancer. Cancer Res. 2020;80(4 suppl):abstr GS6-01. doi:10.1158/1538-7445. SABCS19-GS6-01
9. Chemotherapy administration billing. Noridian Healthcare Solutions. Accessed January 2, 2020. https://med.noridianmedicare.com/web/jea/ topics/drugs-biologicals-injections/chemotherapy-administration-billing

10. Taxol injection. Prescribing information. Bristol-Myers Squibb; 2011 . Accessed December 11, 2020. https://www.accessdata.fda.gov/drugsatfda_docs/label/2011/020262s049lbl.pdf

11. Abraxane. Prescribing information. Celgene; 2012. Accessed December 11, 2020. https://www.accessdata.fda.gov/drugsatfda_docs/label/2012/021660s031lbl.pdf

12. Physician fee schedule search. CMS. Accessed January 3, 2020. https:// www.cms.gov/apps/physician-fee-schedule/search/search-criteria.aspx

13. Clinical laboratory fee schedule. CMS. Accessed January 3, 2020 https://www.cms.gov/Medicare/Medicare-Fee-for-Service-Payment/ ClinicalLabFeeSched

14. Laboratory pricing. Sparrow Health System. Accessed January 3, 2020 https://www.sparrow.org/upload/docs/MySparrow/Laboratory\%20 Pricing.pdf

15. 2020 ASP drug pricing files. CMS. Accessed January 7, 2020. https:// www.cms.gov/medicare/medicare-part-b-drug-average-salesprice/2020-asp-drug-pricing-files

16. Krus GB, Amonkar MM, Smith G, Skonieczny DC, Stavrakas S. Analysis of costs associated with administration of intravenous single-drug therapies in metastatic breast cancer in a U.S. population. J Manag Care Pharm. 2008;14(9):844-857. doi:10.18553/jmcp.2008.14.9.844

17. Bly CA, Molife C, Brown J, et al. The budget impact of including necitumumab on the formulary for first-line treatment of metastatic squamous non-small cell lung cancer: U.S. commercial payer and Medicare perspectives. J Manag Care Spec Pharm. 2018;24(6):534-543. doi:10.18553/jmcp.2018.24.6.534

18. Consumer Price Index databases. U.S. Bureau of Labor Statistics. Accessed January 7, 2020. https://www.bls.gov/cpi/data.htm

19. Bassan F, Peter F, Houbre B, et al. Adherence to oral antineoplastic agent by cancer patients: definition and literature review. Eur J Cancer Care (Engl). 2014;23(1):22-35. doi:10.1111/ecc.12124

20. Aisner J. Overview of the changing paradigm in cancer treatment: oral chemotherapy. Am J Health Syst Pharm. 2007;64(9 suppl 5):S4-S7. doi:10.2146/ajhp070035

21. Cassidy J, Douillard J-Y, Twelves C, et al. Pharmacoeconomic analysis of adjuvant oral capecitabine vs intravenous 5-FU/LV in Dukes' C colon cancer: the X-ACT trial. Br J Cancer. 2006;94(8):1122-1129. doi:10.1038/ sj.bjc.6603059

22. Le Lay K, Myon E, Hill S, et al. Comparative cost-minimisation of oral and intravenous chemotherapy for first-line treatment of non-small cell lung cancer in the UK NHS system. Eur J Health Econ. 2007;8(2):145-151 doi:10.1007/s10198-006-0034-1

VISIT HTTP://BIT.LY/3MMFOWP FOR PDF VISIT HTTP://BIT.LY/38U148T FOR EAPPENDIX 


\title{
The Untapped Potential of Cell and Gene Therapy
}

\author{
LARRY HANOVER
}

EXCITEMENT TOOK WING in the scientific community in the early 1990s, when the first gene therapy trial showed significant success, only to crash at the end of the decade with a patient's tragic death.

More than 20 years later, the excitement is back and greater than before. Although safety remains a concern, investigators are breaking ground in cell and gene therapy, and many believe that ultimately, a string of cancer cures will follow.

"We can absolutely cut the number of cancer deaths down so that one day in our lifetimes it can be a rare thing for people to die of cancer," said Patrick Hwu, MD, president and CEO of Moffitt Cancer Center in Florida and among gene therapy's pioneers. "It still may happen here and there, but it'll be kind of like people dying of pneumonia. It's like, 'He died of pneumonia? That's kind of weird.' I think cancer can be the same way."

The excitement returned in spades in 2017 when the FDA signed off on a gene-therapy drug for the first time, approving the chimeric antigen receptor (CAR) T-cell treatment tisagenlecleucel (Kymriah; Novartis) for the treatment of Bcell precursor acute lymphoblastic leukemia. At last, scientists had devised a way to reprogram a person's own T cells to attack tumor cells.

"We're entering a new frontier," said Scott Gottlieb, MD, then the FDA Commissioner, in announcing the groundbreaking approval.

Gottlieb wasn't exaggerating. The growth in CAR T-cell treatments is exploding. Although only a handful of cell and gene therapies are on the market, FDA officials predicted in 2019 that the agency will receive more than 200 investigational new drug applications per year for cell and gene therapies, and that by 2025, it expects to have accelerated to 10 to 20 cell and gene therapy approvals per year.

"Essentially, you can kill any cancer cell that has an antigen that is recognized by the immune cell," Hwu said. "The key to curing every single cancer, which is our goal, is to have receptors that can recognize the tumor but don't recognize the normal cells." Receptors recognizing and then attacking normal cells is what can cause toxicity.

Cell therapy involves cultivating or modifying immune cells outside the body before injecting them into the patient. Cells may be autologous (self-provided) or allogeneic (donor-provided); they include hematopoietic stem cells and adult and embryonic stem cells. Gene therapy modifies or manipulates cell expression. There is considerable overlap between the 2 disciplines.

Juliette Hordeaux, PhD, senior director of translational research for the University of Pennsylvania's gene therapy program, is cautious about the FDA's predictions, saying she'd be "thrilled" with 5 cell and/or gene therapy approvals annually.

"For monogenic diseases, there are only a certain number of mutations, and then we'll plateau until we reach a stage where we can go after more common diseases," Hordeaux said.

"Safety has been the main brake around adeno-associated virus vector (AAV) gene therapy," added Hordeaux, whose hospital's program has the institutional memory of both Jesse Gelsinger's tragic death during a 1999 gene therapy trial as well as breakthroughs by Carl June, MD, and others in CAR T-cell therapy. "Sometimes there are unexpected toxicity [events] in trials....I think figuring out ways to make gene therapy safer is going to be the next goal for the field before we can even envision many more drugs approved."

In total, 3 CAR T-cell therapies are now on the market, all targeting the CD19 antigen. Tisagenlecleucel was the first. Gilead
Sciences received approval in October 2017 for axicabtagene ciloleucel (axi-cel; Yescarta), a CAR T-cell therapy for adults with large B-cell non-Hodgkin lymphoma. Kite Pharma, a subsidiary of Gilead, received an accelerated approval in July 2020 for brexucabtagene autoleucel (Tecartus) for adults with relapsed or refractory mantle cell lymphoma.

On February 5, 2021, the FDA approved another CD19-directed therapy for relapsed/refractory large B-cell lymphoma, lisocabtagene maraleucel (liso-cel; JCAR017; Bristol Myers Squibb). The original approval date was missed due to a delay in inspecting a manufacturing facility (see related article, SP52).

Idecabtagene vicleucel (ide-cel; bb2121; Bristol Myers Squibb) is under priority FDA review, with a decision expected by March 31,2021 . The biologics license application seeks approval for ide-cel, a B-cell maturation antigen-directed CAR therapy, to treat adult patients with multiple myeloma who have received at least 3 prior therapies.

The number of clinical trials evaluating CAR T-cell therapies has risen sharply since 2015, when investigators counted a total of 78 studies registered on the ClinicalTrials.gov website. In June 2020, the site listed 671 trials, including 357 registered in China, 256 in the United States, and 58 in other countries. ${ }^{3}$

Natural killer (NK) cells are the research focus of Dean Lee, MD, $\mathrm{PhD}$, a physician in the Division of Hematology and Oncology at Nationwide Children's Hospital. He developed a method for consistent, robust expansion of highly active clinical-grade NK cells that enables repeated delivery of large cell doses for improved efficacy. This finding led to several first-in-human clinical trials evaluating adoptive immunotherapy with expanded NK cells under an FDA Investigational New Drug application. He is developing both genetic and nongenetic methods to improve tumor targeting and tissue homing of NK cells. His efforts are geared toward pediatric sarcomas

"The biggest emphasis over the past 20 to 25 years has been cell therapy for cancer, talking about trying to transfer a specific part of the immune system for cells," said Lee, who is also director of the Cellular Therapy and Cancer Immunology Program at Nationwide Children's Hospital, at The Ohio State University Comprehensive Cancer Center - Arthur G. James Cancer Hospital, and at the Richard J. Solove Research Institute.

The Pivot Toward Treating COVID-19 and Other Diseases However, Lee said, NKs have wider potential. "This is kind of a natural swing back. Now that we know we can grow them, we can reengineer them against infectious disease targets and use them in that [space]," he said.

Lee is part of a coronavirus disease 2019 (COVID-19) clinical trial, partnering with Kiadis, for off-the-shelf K-NK cells using Kiadis' proprietary platforms. Such treatment would be a postexposure preemptive therapy for treating COVID-19. Lee said the pivot toward treating COVID-19 with cell therapy was because "some of the very early reports on immune responses to coronavirus, both original [SARS-CoV-2] and the new [mutation], seem to implicate that those who did poorly [overall] had poorly functioning NK cells."

The revolutionary gene editing tool CRISPR is making its initial impact in clinical trials outside the cancer area. Its developers, Jennifer Doudna, $\mathrm{PhD}$, and Emmanuelle Charpentier, $\mathrm{PhD}$, won the Nobel Prize in Chemistry 2020. "

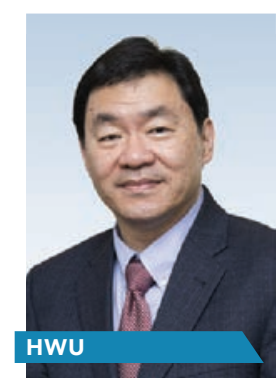

Patrick $H w u, M D$, president and $C E O$ of Moffitt Cancer Center in Florida

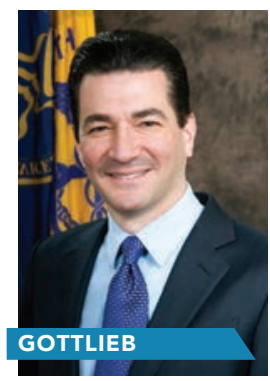

Scott Gottlieb, MD, former FDA commisioner

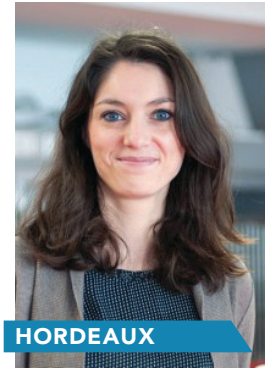

Juliette Hordeaux, $P h D$, senior direct of translational research, University of Pennsylvania Gen Therapy Program

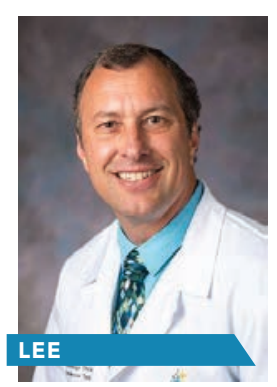

Dean Lee, MD, PhD, physician in the Divisio of Hematology and Oncology at Nationwide Children's Hospital 


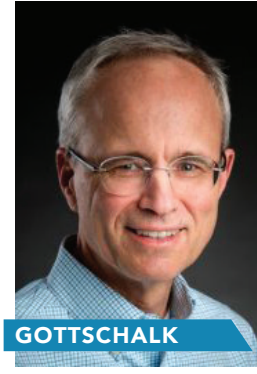

Stephen Gottschalk $M D$, chair of the department of Bone Marrow Transplantation \& Cellular Therapy at St Jude Children Research Hospital
For patients with sickle cell disease (SCD), CRISPR was used to reengineer bone marrow cells to produce fetal hemoglobin, with the hope that the protein would turn deformed red blood cells into healthy ones. National Public Radio did a story on one patient who, so far, thanks to CRISPR, has been liberated from the attacks of SCD that typically have sent her to the hospital, as well from the need for blood transfusions. ${ }^{4}$

"It's a miracle, you know?" the patient, Victoria Gray of Forest, Mississippi, told NPR.

She was among 10 patients with SCD or transfusion-dependent beta-thalassemia treated with promising results, as reported by the New England Journal of Medicine. ${ }^{5}$ Two different groups, one based in Nashville, which treated Gray, ${ }^{5}$ and another based at Dana-Farber Cancer Institute in Boston, ${ }^{6}$ have reported on this technology.

Stephen Gottschalk, MD, chair of the department of bone marrow transplantation and cellular therapy at St Jude Children's Research Hospital, said, "There's a lot of activity to really explore these therapies with diseases that are much more common than cancer."

Animal models use $\mathrm{T}$ cells to reverse cardiac fibrosis, for instance, Gottschalk said. Using T cells to reverse pathologies associated with senescence, such as conditions associated with inflammatory clots, are also being studied.

Hordeaux said she foresees AAV being used more widely to transmit neurons to attack neurodegenerative diseases.

"The neurons are easily transduced by AAV naturally," she said. "AAV naturally goes into neurons very efficiently, and neurons are long lived. Once we inject genetic matter, it's good for life, because you don't renew neurons."

\section{Logistical Issues}

Speed is of the essence, as delays in producing therapies can be the difference between life and death, but the approval process takes time. The process of working out all kinks in manufacturing also remains a challenge. Rapid production is difficult, too, because of the necessary customization of doses and the need to ensure a safe and effective transfer of cells from the patient to the manufacturing center and back into the patient.

Other factors that can slow down launches include insurance coverage, site certification, staff training, reimbursement, and patient identification. The question of how to reimburse has not been definitively answered; at this point, insurers are being asked to issue 6- or even 7-figure payments for treatments and therapies that may not work. ${ }^{8}$

"CAR T, I think, will become part of the standard of care," Gottschalk said. "The question is how to best get that accomplished. To address the tribulations of some autologous products, a lot of groups are working with off-the-shelf products to get around some of the manufacturing bottlenecks. I believe those issues will be solved in the long run."

\section{REFERENCES}

1. Statement from FDA Commissioner Scott Gottlieb, MD, and Peter Marks, MD, PhD, director of the Center for Biologics Evaluation and Research on new policies to advance development of safe and effective cell and gene therapies. News release. FDA website. January 15, 2019. https://www.fda.gov/news-events/press-announcements/statement-fda-commissioner-scott-gottlieb-md-and-peter-marks-md-phd-director-center-biologics. Accessed January 13, 2021

2. Bristol Myers Squibb provides regulatory update on lisocabtagene maraleucel (liso-cel). News release. Bristol Myers Squibb; November 16, 2020. Accessed January 11, 2021. https://news. bms.com/news/details/2020/Bristol-Myers-Squibb-Provides-Regulatory-Update-on-Lisocabtagene-Maraleucel-liso-cel/default.aspx

3. Wei J, Guo Y, Wang Y. et al. Clinical development of CAR T cell therapy in China: 2020 update. Cell Mol Immunol. Published online September 30, 2020. doi:10.1038/s41423-020-00555-x

4. Stein R. CRISPR for sickle cell diseases shows promise in early test. Public Radio East. November 19, 2019. Accessed January 11, 2021. https://www.publicradioeast.org/post/crisprsickle-cell-disease-shows-promise-early-test

5. Frangoul H, Altshuler D, Cappellini MD, et al. CRISPR-Cas9 gene editing for sickle cell disease and $\beta$-Thalassemia. $N$ Engl J Med. Published online December 5, 2020. DOI: 10.1056/ NEJMoa2031054

6. Esrick EB, Lehmann LE, Biffi A, et al. Post-transcriptional genetic silencing of BCL11A to treat sickle cell disease. N Engl J Med. Published online December 5, 2020. doi:10.1056/ NEJMoa2029392

7. Yednak C. The gene therapy race. PwC. February 5, 2020. Accessed January 11, 2021. https:// www.pwc.com/us/en/industries/health-

industries/library/gene-therapy-race.html

8. Gene therapies require advanced capabilities to succeed after approval. PwC website Accessed January 11, 2021. https://www.pwc.com/us/en/industries/health-industries/ library/commercializing-gene-therapies.html

\title{
Tanya Siddiqi, MD, Discusses the Promise of Reduced Toxicity With Liso-Cel
}

\author{
MARY CAFFREY AND ROSE MCNULTY
}

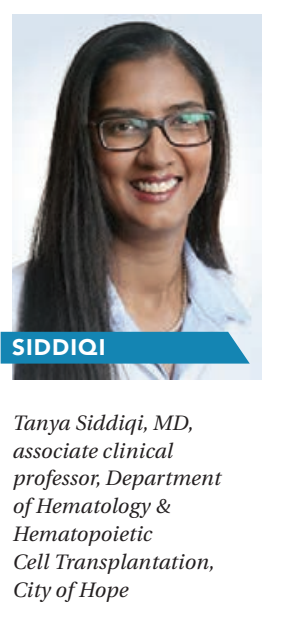

MORE THAN 3 YEARS after the FDA approved the first options in chimeric antigen receptor (CAR) T-cell therapy, Bristol Myers Squibb (BMS) and Juno Therapeutics received word February 5, 2021, that lisocabtagene maraleucel (liso-cel) was approved for the treatment of relapsed or refractory (R/R) large B-cell lymphoma, following progression after at least 2 prior therapies. ${ }^{1}$

The approval for liso-cel, to be sold as Breyanzi, followed a lengthy approval process that saw the termination of a contingent value rights (CVR) agreement. That deal would have paid investors if the FDA approved liso-cel by December 31, 2020. On November 16,2020 , BMS announced that the FDA would not meet its target approval date due to the agency's inability to complete inspections at a third-party manufacturing site, because of travel restrictions related to the coronavirus disease 2019 (COVID-19) pandemic. $^{2}$
Like its predecessors, liso-cel targets CD19, a surface glycoprotein seen in B-cell development that remains present when the cells become malignant. According to information from BMS, the liso-cel CAR mechanism features the following:

- an anti-CD19 single-chain variable fragment targeting domain for antigen specificity

- a transmembrane domain

- a 4-1BB costimulatory domain intended to increase T-cell proliferation and persistence

- a CD3-zeta T-cell activation domain

In addition, liso-cel's distinct manufacturing process creates a defined composition of CD8+ and CD4+ T cells, which may reduce product variability; however, the drugmaker states, 
"The clinical significance of defined composition is unknown."

During the JP Morgan conference on January 11, 2021, BMS CEO Giovanni Caforio, MD, said he was "confident" in the future of liso-cel as a "differentiated" CAR T-cell therapy. ${ }^{4}$ Along with the approval, BMS launched Cell Therapy 360, an online program that gives updates for patients and physicians on when they can expect their CAR T therapy to arrive to their treatment center, along with support services. Patients treated with liso-cel will be able to monitor their condition post-infusion with disposable wearable technology connected to a smartphone, according to an email from BMS.

For insights on what the arrival of liso-cel could mean in the treatment landscape, Evidence-Based Oncology $^{\mathrm{TM}}(E B O)$ turned to Tanya Siddiqi, MD, director of the chronic lymphocytic leukemia program at Toni Stephenson Lymphoma Center and associate clinical professor, Department of Hematology \& Hematopoietic Cell Transplantation, at City of Hope in Duarte, California.

Siddiqi was an investigator for ZUMA- $1,{ }^{5}$ which led to the approval of axicabtagene ciloleucel (axi-cel), sold as Yescarta, and the TRANSCEND NHL trial ${ }^{6}$ for liso-cel. She has addressed major scientific meetings on the challenge of managing the toxicities associated with CAR T-cell therapy - and discussed how liso-cel represents a step forward over its predecessors.

This interview, conducted before the BMS announcement, has been edited for clarity and length.

AJMC ${ }^{\circledR}$ : We're anticipating an FDA decision on liso-cel before the end of the year. Can you discuss the need of the patients who would take this new CAR T-cell therapy?

SIDDIOI: For CAR T-cell therapy targeting CD19positive B-cell lymphomas-specifically aggressive B-cell lymphomas-we already have a couple of FDA-approved options. The question is: What is liso-cel? How is it different? Why would people pick this over other things? In the trials that we've conducted, we found that liso-cel seems to have lesser toxicity in terms of the specific CAR T-cell adverse effects of cytokine release syndrome (CRS) or hyperinflammation, as well as neurotoxicity. We've just seen fewer severe adverse events so much so that at some [cancer] centers across the country, we've been able to give liso-cel CAR T cells to patients in the clinic or outpatient setting rather than having to admit them to the hospital, depending on the patient's situation.

Those are the strengths of liso-cel-less toxicity and thus, a better chance of giving it in the outpatient setting with hospital admission available to anyone who develops a fever or other advese effects. This means fewer days of inpatient hospitalization for these patients, so it may be less costly overall. I don't think the efficacy is necessarily differentmeaning that it seems to work as well as the other FDA-approved products already commercially available. But for the reasons that I've listed, I think it might be a very good option for older patients, maybe patients who are bit more frail, or younger patients who just don't want to be admitted to the hospitalthey just want to try to do it in the outpatient setting.
EBO: You touched on this already, but can you discuss how Iiso-cel differs from earlier CAR T-cell therapies-both in the way it's manufactured and how it works, and what that reduced variability means for patients?

SIDDIQI: Liso-cel is manufactured in a way that it gives very precise, equal numbers of CAR $\mathrm{T}$ cells that are labeled CD4 and CD8, in a 1:1 ratio. All of us have $\mathrm{T}$ cells to fight infections with, and these $\mathrm{T}$ cells are what we take from patients. Then, we modify them in the lab by genetic engineering in order to produce CAR T cells so that now instead of looking for infections, these CAR T cells are going to look for B-cell lymphoma cells and fight lymphoma.

\section{"We always try to advise our referring physicians, and educate patients, at conferences, to try to send these patients to us before they are at the end of the line-before they've tried and failed everything, and now there's just rampant disease. [At that point,] you're dealing with a situation where the patient is going to have more side effects and will not be able to tolerate the CAR T cells as well." \\ - Tanya Siddiqi, MD, associate clinical professor,} Transplantation, City of Hope

The other products are given back to patients as a bag of CAR T cells mixed with potentially varying ratios of different types of T cells-CD4+, CD8+, etc. With liso-cel, the manufacturing process actually separates out the $\mathrm{CD} 4+$ and $\mathrm{CD} 8+$ types of T cells first, and then manufactures CAR T cells out of them separately. So when we give the cells back to patients, we give it in a 1:1 ratio of CD4+ and CD8+ cells. We know exactly how many CD4+ and how many CD8+ $\mathrm{T}$ cells these patients receive. And the thought is, the researchers and the drug manufacturer feel that this helps to have an expectation of what expansion you will have of these cells in the body.

Therefore, we potentially have an idea of what type of side effects or how severe the side effects might be. It may limit some of those side effects, or at least make them a little bit more predictable or controlled.

EBO: That's a great way to shift to your own work on length of stay due to CRS. What do we know about the key variables in determining whether a patient will experience an adverse effect that requires an extended stay in the hospital, and can more be done to avoid lengthy hospital stays?

SIDDIOI: That's a very important question. Because lengthy hospital stays, especially in the [intensive care unit], really add to the bill and the financial burden of these treatments. We know that people who have a big burden of disease going into CAR T-cell therapy, meaning they have a lot of lymphoma in their bodies, they tend to be at higher risk for more side effects like CRS and neurotoxicity, probably because there's so much inflammation that's generated while these CAR T cells are trying to fight the lymphoma. What we know is that people who come to us for CAR T cells with lesser disease might have fewer side effects potentially and a better overall outcome.

So we always try to advise our referring physicians, and educate patients, at conferences, to try to send these patients to us before they are at the end of the line-before they've tried and failed everything, and now there's just rampant disease. [At that point,] you're dealing with a situation where the patient is going to have more adverse effects and will not be able to tolerate the CAR T cells as well. Instead, if they fail two lines of therapy and the disease is still small in volume, but it's starting to progress, we can treat them more effectively with CAR T cells and with fewer side effects potentially.

EBO: That brings up the next topic: There have been discussions that CAR T-cell therapy should be given earlier during treatment. As you said, if it's not given as the last resort, patients might respond better. Where do you see those patterns heading in the future? And would that be truer for some patients than others?

SIDDIOI: With aggressive diffuse large B-cell lymphoma, there's about a $60 \%$ to $70 \%$ chance of curing that in the frontline setting. With the line of chemoimmunotherapy, you can cure $60 \%$ to $70 \%$ of patients so that it never comes back. But the rest of them-when it just relentlessly keeps coming back and it's hard to cure-once those patients relapse they tend to keep relapsing. So our mainstay in the relapse setting is to give them salvage chemoimmunotherapy, collect stem cells, and take them to autologous stem cell transplantation if they've achieved a remission with the salvage chemotherapy. If they haven't achieved remission with that salvage chemotherapy, then they should go on to CAR T-cells directly instead of waiting and trying more and more chemotherapies. After failing second-line therapy, the FDA approval allows us to try CAR T-cells. There are studies that are now ongoing that are comparing CAR T-cells to autologous stem cell transplantation after failing first-line therapy. So once patients relapse the first time, these studies are comparing giving them salvage chemotherapy and transplant, versus taking them straight to CAR T-cells. Once we have that data, we'll know better whether we can do CAR T-cells even earlier in the lines of therapy.

EBO: We've been hearing for some time more about allogeneic or off-the-shelf therapies. What progress has been made on in that technology?

SIDDIOI: I'm not too involved with these trials myself, but I know we have trials at City of Hope that are ongoing with off-the-shelf therapy. What I can tell you is that it's very attractive in that you don't have to collect T-cells from patients, keeping their " 
lymphoma under control while these $\mathrm{T}$ cells then go to the lab and CAR T-cells are manufactured in 2 to 4 weeks depending on which product it is, and then they come back and get infused. With off-the-shelf products, you can just grab it and go as soon as you know the patient needs it.

The initial concerns were because the cells are not from the patient themselves- the cells are from donors. Across the board there might be concerns of rejection and what's called graft-versus-host disease and things like that. So far, I don't think in the trial they've come up with such adverse effects to any significant extent. What I don't know is whether they've come up with a good result yet. Is it looking like the benefits of taking off-the-shelf CAR T-cells are as good as autologous CAR T cells, meaning patients' own CAR T cells? I think that remains to be seen. If they are, then it's much easier to use off-theshelf CAR T cells.

EBO: How is COVID-19 affecting the clinical trial process for CAR T-cell therapy?

SIDDIOI: When the pandemic kind of started surging early in the year, and when we went into lockdown mode from March onward, we and other centers across the country took a lot of steps to slow down our clinical trial enrollment. Our staff started staggering who would come into work which day of the week and who could work from home. For those in the clinical trials office, there was a lot of need for safety and logistical reasons for us to slow down enrollment onto clinical trials. And there were other questions, such as, who would take care of patients at home once we discharged them after they received CAR T cells? What if their caregivers were exposed and got sick? Logistically, it was difficult to safely do many trials, especially CAR T-cell trials and transplants earlier in the year.

Since the end of summer, we ramped up again, and we're now doing as many transplants and CAR T cells as we were probably doing last year.
So, we're pretty much all the way up again, but I don't know how this winter will go because COVID is surging again.

As far as just CAR T cells themselves, we had to also think about travel for the cells because Juno Therapeutics is in Seattle, and Kite Pharma is here in Los Angeles, but Novartis is elsewhere. Just the movement of these cells was a concern because of travel restrictions during COVID-19. But as far as I know, the companies did not lose that commitment They told us, we'll get the cells to you, we will find a way to do it. I don't think any patients went without cells who should have received cells.

EBO: What advice do you have for community oncologists interested in CAR T-cell therapy for their patients?

SIDDIOI: There's good news for community physicians. We may soon have a therapeutic option of liso-cel CAR T-cell therapy which seems to have lesser adverse effects. So, this might make things cheaper due to less need for hospitalization potentially without compromising the chance of cure. We want these patients to try CAR T-cell therapy sooner rather than later in their relapses. You can always try multiple cycles of chemotherapy at some other time if you relapse again, but if you can be cured with CAR $T$ cells such that you never need treatment again, why not try that first? For the patients who respond well to CAR T cells, the treatment works extremely well. And that's the holy grail: to find the cure for all patients.

Maybe only half the patients will currently have a very good and durable response- - but those patients may never relapse again. So why not try it sooner rather than later? And of course, we're always looking for trial patients, because now we need to improve these results even further. So, community oncologists should also refer for trials, because I think that it's very important to have trials with different combinations-CAR T cells plus another immunotherapy agent-to see if we can improve upon the response rates even more.

REFERENCES

1. FDA approves new treatment for adults with relapsed or refractory large B-cell lymphoma. News release. Food and Drug Administration February 5, 2021. https://www.prnewswire.com/news-releases/fdaapproves-new-treatment-for-adults-with-relapsed-or-refractory-largeb-cell-lymphoma-301223275.htm Bristol Myers Squibb provides regulatory update on lisocabtagene maraleucel (liso-cel). News release. Business Wire. November 16, 2020. Accessed January 11, 2021. https://www.businesswire.com/news/

home/20201116006018/en/Bristol-Myers-Squibb-Provides-RegulatoryUpdate-on-Lisocabtagene-Maraleucel-liso-cel

3. Bristol Myers Squibb provides update on biologics license application (BLA) for lisocabtagene maraleucel (liso-cel). News release. Bristol Myer Squibb. May 6, 2020. Accessed January 11, 2021. https://news.bms.com/ news/details/2020/Bristol-Myers-Squibb-Provides-Update-onBiologics-License-Application-BLA-for-Lisocabtagene-Maraleucelliso-cel/default.aspx\#: :text=Liso\%2Dcel\%20aims\%20to\%20target,a\%20 CD3\%2Dzeta\%20T\%2Dcell

4. Liu A. JPM: Deal-making still a 'top-priority' at Bristol Myers as CEO eyes midsize buys. FiercePharma. January 11, 2011. Accessed January 11, 2021. https://www.fiercepharma.com/pharma/jpm-21-m-a-still-a-toppriority-at-bristol-myers-as-ceo-eyes-midsize-deals

5. Neelapu SS, Locke FL, Bartlett NL et al. Axicabtagene ciloleucel CAR T-cell therapy in refractory large B-cell lymphoma. $N$ Engl J Med. 2017;377(26):2531-2544. doi:10.1056/NEJMoa1707447

6. Abramson JS, Palomba ML, Gordon LI, et al. Lisocabtagene maraleucel for patients with relapsed or refractory large B-cell lymphomas (TRANSCEND NHL 001): a multicentre seamless design study. Lancet. 2020;396(10254):839-852. doi:10.1016/S0140-6736(20)31366-0

\section{Targeted i.: Oncology}

Ide-cel CAR T-Cell Therapy Demonstrates Promise in Heavily Pretreated Myeloma Read more at: http://bit.ly/3iSnGEx

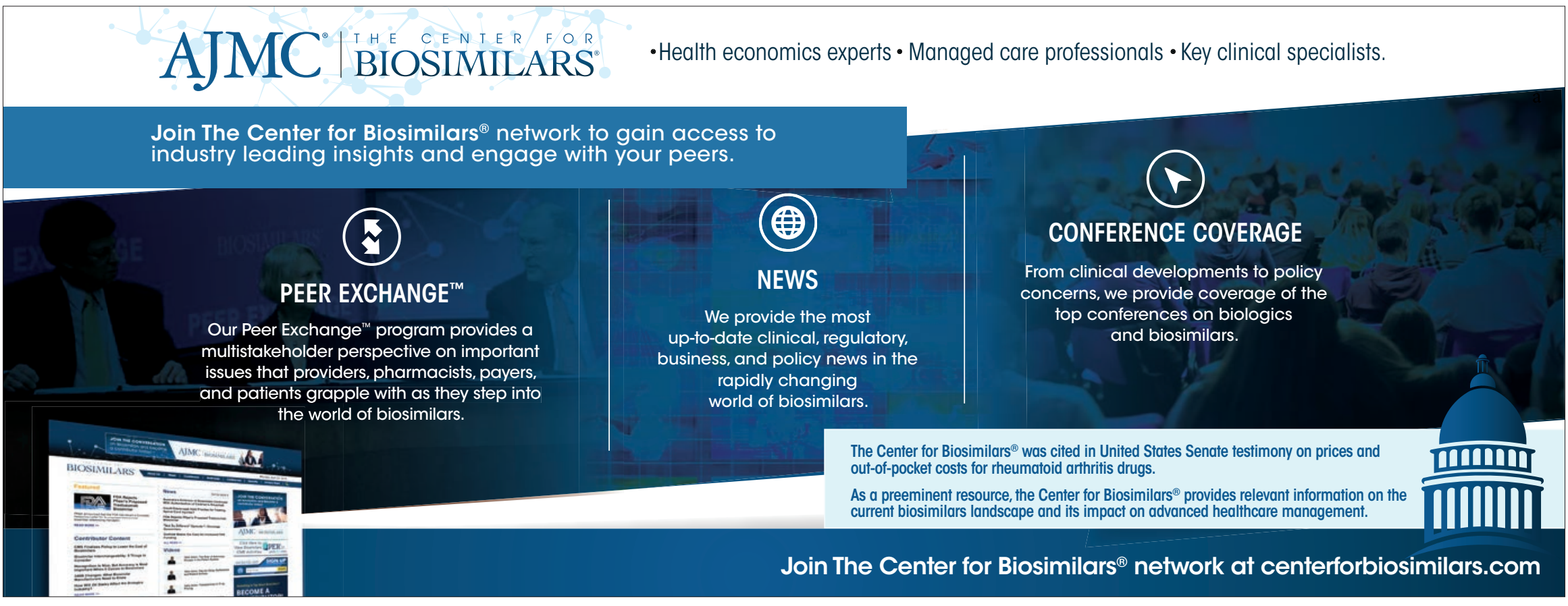




\title{
FDA Approves Osimertinib for Adjuvant Treatment of Early-Stage EGFR-Mutated NSCLC
}

\author{
ROSE MCNULTY
}

THE FDA HAS APPROVED OSIMERTINIB (Tagrisso) for the adjuvant treatment of patients with early-stage non-small cell lung cancer (NSCLC) with epidermal growth factor receptor-mutated $(E G F R m)$ tumors. ${ }^{1}$ This marks the first FDA approval of an adjuvant treatment for NSCLC with EGFR exon 19 deletions or exon 21 L858R mutations.

The December 18, 2020, approval was based on results from the phase 3 ADAURA trial, in which osimertinib showed significant improvement in disease-free survival (DFS) in patients with stage II to IIIA NSCLC with EGFRm tumors and in the overall trial population of stage IB to IIIA EGFRm NSCLSC. ${ }^{2}$ The review was conducted under the FDA Oncology Center of Excellence's Project Orbis, which allows concurrent submission and review of oncology drugs among international partners.

In the double-blind, phase 3 trial, patients with resected EGFRm NSCLC $(n=682)$ were randomized to receive either $80 \mathrm{mg}$ of osimertinib $(n=339)$ orally once daily or a placebo $(n=343)$ after recovery from surgery with or without standard adjuvant chemotherapy for 3 years. The primary end point was DFS in stage I to IIIA disease, and secondary end points included DFS in the overall population, overall survival, and safety.

Adjuvant osimertinib reduced the risk of disease or death in patients with stage II to IIIA disease by $83 \%$ (HR, $0.17 ; 99.06 \%$ CI, 0.11-0.26; $P<.001)$ compared with the placebo group. At 24 months, $90 \%$ of the osimertinib cohort was alive and disease-free (95\% CI, 84\%-93\%), compared with $44 \%$ of the placebo group.

Overall, $89 \%$ (95\% CI, 85\%-92\%) of the participants in the study who received osimertinib and 52\% (95\% CI, $46 \%-58 \%$ ) of patients in the placebo group were alive and disease-free at 24 months. The overall $\mathrm{HR}$ for disease recurrence or death was 0.20 for patients receiving osimertinib $(99.12 \% \mathrm{CI}, 0.14-0.30 ; P<.001)$.

"EGFR inhibitors in the advanced setting slow disease recurrence and provide palliation. But there's always resistance that's going to develop. But by using them earlier on, the hope is that we're able to really cure patients. We get to disease when it's at a much lower disease burden and fewer resistant cells," ADAURA investigator Roy S. Herbst, MD, PhD, chief of medical oncology at Yale Cancer Center and Smilow Cancer Hospital, said in an interview.

The ability to utilize osimertinib in the earliest stages of disease is practice changing, Herbst said. He stressed the importance of testing for mutations early on in treatment and said his clinic at Yale will start screening patients and genomic profiling at the time of surgery to identify those who are eligible for adjuvant osimertinib.

Osimertinib has been approved as a first-line therapy for untreated metastatic NSCLS with EGFR exon 19 deletions or exon 21 L858R mutations since 2018, ${ }^{3}$ but this latest indication represents a patient population with a need for treatment options. An estimated $76 \%$ of lung cancer cases are NSCLC, and about $20 \%$ of those patients with have EGFRm NSCLC. In this population, about $30 \%$ have resectable disease and would be eligible for treatment with osimertinib in the adjuvant setting.

"We're still waiting for survival [data] on this trial, but we've shown amazing disease-free survival benefits- $83 \%$ improvement. And we've also shown fewer metastases to vital organs such as the brain," Herbst said. "I think having this option now for patients is just so wonderful, especially a couple of months earlier than expected, and I think it's going to take off very quickly. It was already being used, but of course it's important to have the FDA approval for reimbursement, especially." •

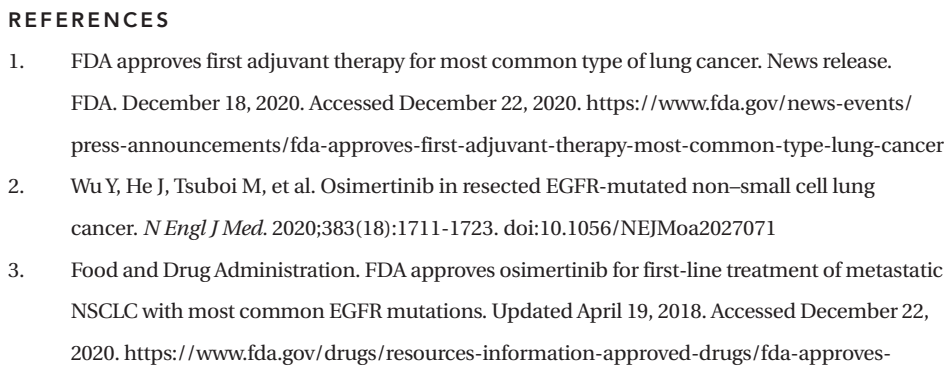

osimertinib-first-line-treatment-metastatic-nsclc-most-common-egfr-mutations

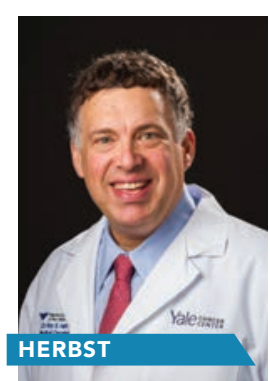

\section{Certain NSCLC Patients Are More Likely to Respond to Immunotherapy, Study Results Show}

\author{
ROSE MCNULTY
}

IMMUNOTHERAPY CAN BE an important tool in cancer treatment, but as with most therapies, its effectiveness varies among patients for reasons that are not always clear. When it comes to non-small cell lung cancer (NSCLC), a study published in Nature investigated why NSCLC patients with a type of human leukocyte antigen (HLA) called B44 were not more likely to respond to immunotherapy compared with patients lacking HLA-B44, when the opposite is generally true in melanoma. ${ }^{1}$
About half of the population has HLA-B44, and those people typically do well on immunotherapy for melanoma. But researchers at the UCLA Jonsson Comprehensive Cancer Center noticed that the same did not hold true in NSCLC, prompting them to investigate the mechanisms leading to the surprising finding. They chose B44 over other HLA-B supertypes because of its common occurrence, which allows for larger cohorts and therefore more statistically significant conclusions.

\begin{abstract}
"Usually you would think that for 2 types of cancers that generally respond well to immunotherapy, there would be similar principles in terms of characteristics of patients who benefit, but that's not the case in this instance," Amy Cummings, MD, clinical instructor of hematology/oncology at the David Geffen School of Medicine at UCLA and member of the Jonsson Cancer Center, said in a press release. ${ }^{2}$

Researchers aimed to build on a previous study that found HLA-B44 presence did not benefit "
\end{abstract}




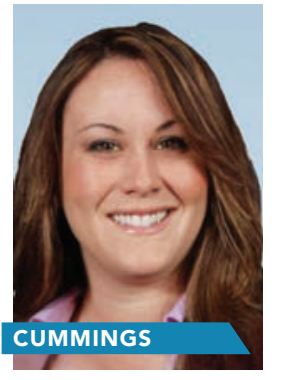

Amy Cummings, $M D$, clinical instructor of hematology/oncology at the David Geffen School Medicine at UCLA

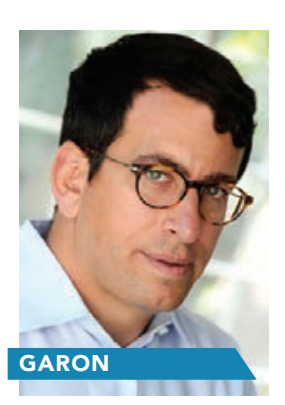

Edward Garon, $M D, M S$, director of the UCLA Jensson Cancer Center signal transduction and
therapeutics program
NSCLC patients the way it did melanoma patients. The new study analyzed a group of patients treated with pembrolizumab with more than 5 years of follow-up and available tissue samples to get more data on the connection between HLA-B44 and immunotherapy outcomes.

To find out why patients with HLA-B44 have varied responses to immunotherapy in NSCLC versus melanoma, researchers also evaluated the mechanisms behind the different responses and validated the findings in publicly available NSCLC and melanoma cohorts.

When they performed whole-exome sequencing on melanoma and NSCLC tumors and blood samples, they found that different responses to immunotherapy were associated with the different mutations commonly found in each type of cancer. The presence of somatic mutations that lead to negatively charged glutamic acid anchors were indicators that NSCLC patients would see immunotherapy benefits similar to those seen in melanoma.

They analyzed treatment outcomes based on the neoepitopes, or peptides that the immune system recognizes as abnormal and targets with $\mathrm{T}$ cells, generated by the mutations. They predicted which neoepitopes would most effectively bind to HLA-B44 and be recognized by the immune system, then assessed treatment outcomes, including survival. They found that only NSCLC patients who had similar neoepitopes to those of melanoma patients were significantly responsive to immunotherapy.

When NSCLC patients did have HLA-B44 and the melanoma-like neoepitopes, their responses were typically durable, lasting for years in some cases. That information could help researchers running clinical trials stratify patients in terms of how likely they are to respond to immunotherapy, Cummings said.

"Our analyses offer an opportunity to move beyond histologic associations toward mechanism-informed prognostic markers for patient stratification: while B44, on average, is not predictive of ICB response in NSCLC, it may be for patients with NSCLC who exhibit favorable B44 neoepitopes with the converse true for patients with B44 melanoma without favorable B44 neoepitopes," the authors wrote.

Authors noted that follow-up length varied and the study size (65 participants) was limited, but they concluded that the presence of certain neoepitopes does suggest immune tolerance and immunotherapy outcomes, which might explain the inconsistencies previously seen in immunotherapy response in patients with HLA-B44.

"From the time that we discovered the contradictory outcomes in HLA-B44 patients with melanoma and non-small cell lung cancer, we became fascinated by the mechanism that could explain this," senior author Edward Garon, MD, MS, professor of hematology/oncology and director of the signal transduction and therapeutics program at the Jonsson Cancer Center, said in a statement. "While seeking this explanation, we gained important insight into how the immune system identifies tumors. We hope to eventually harness these findings to design therapies that can further enhance the immune response against tumors in specific patients." •

\section{REFERENCES}

1. Cummings AL, Gukasyan J, Lu HY, et al. Mutational landscape influences immunotherapy outcomes among patients with non-small cell lung cancer with human leukocyte antigen supertype B44. Nature. Published online November 16, 2020. Accessed December 2, 2020. doi:10.1038/s43018-020-00140-1

2. Study identifies patients with lung cancer most likely to respond to immunotherapy. News release. UCLA Jonsson Comprehensive Cancer Center. November 16, 2020. Accessed December 2, 2020. https://cancer.ucla.edu/Home/Components/News/News/1590/1631

\section{Possible Treatment Identified for Aggressive NSCLC Subset}

Rose McNulty

A POTENTIAL TREATMENT for patients with non-small cell lung cancer (NSCLC) with KRAS and LKB1 mutations was discovered by researchers from the Children's Medical Center Research Institute (CRI) at the University of Texas Southwestern (CRI). ${ }^{1}$ Tumors with both mutations, known as KL tumors, are highly aggressive, and patients who have them usually do not respond to immunotherapy.

Individually, KRAS and LKB1 mutations can alter a patient's metabolism and response to treatment. However, when both genes are mutated in the same tumor, the exact ways in which they promote tumor growth and metastasis are not yet clear. But the recent study from the CRI found that inhibiting the hexosamine biosynthesis pathway (HBP), which allows cells to modify proteins, could be a potential treatment in those types of tumors. ${ }^{2}$

"We used to think that most tumors rely on the same handful of metabolic pathways to grow, but we've learned over the last decade that this is an oversimplification. Instead, different tumor subclasses have particular metabolic needs arising from mutations in key genes," study author Ralph DeBerardinis, MD, PhD, a professor at CRI and a Howard Hughes Medical Institute investigator, said in a statement. "Understanding how specific combinations of mutations promote tumor growth and metastasis may allow us to design tailored therapies for patients."

In the study that led to the findings, published in Nature Metabolism, the researchers compared the metabolic properties of genetically engineered $\mathrm{KL}$ tumors in mice with tumors with different mutations and with a normal lung They found that in KL tumors, the HBP was activated. This is in line with previous research from the Ralph DeBerardinis Laboratory showing that $K L$ cells reprogram carbon and nitrogen metabolism, promoting growth but increasing their sensitivity to certain inhibitors. ${ }^{3}$

$\mathrm{KL}$ tumor growth is fueled by a high rate of protein production, which is thought to require $\mathrm{HBP}$ activation. To inhibit that pathway, researchers targeted GFPT2, which they identified as a liability in KL tumors. Targeting GFPT2 did not have much effect on tumors with KRAS mutations alone, but it did suppress $\mathrm{KL}$ tumor growth in the mouse models. These findings suggest that GFPT2 could be a useful target in NSCLC with KRAS and LKB1 mutations.
Currently, there is no GFPT2 inhibitor, so researchers aim to look into blocking steps along the pathway, said Jiyeon Kim, PhD, the postdoctoral fellow who led the study with DeBerardinis.

"Since no specific inhibitor against GFPT2 exists, our next step is to see if blocking certain steps in the glycosylation pathway could be therapeutically beneficial," Kim said. "Ultimately we are looking for options that can help stop the growth and spread of these aggressive tumors." •

REFERENCES

1. Researchers uncover a potential treatment for an aggressive form of lung cancer. News release. Children's Medical Center Research Institute at UT Southwestern; January 5, 2021. Accessed January 8, 2021. https://www.utsouthwestern.edu/newsroom/ articles/year-2021/aggressive-form-of-lung-cancer.html

1. Kim J, Lee HM, Cai F, et al. The hexosamine biosynthesis pathway is a targetable liability in KRAS/LKB1 mutant lung cancer. Nat Metab. Published online November 30, 2020. doi:10.1038/ s42255-020-00316-0

1. Kim J, Hu Z, Cai L, et al. CPS1 maintains pyrimidine pools and DNA synthesis in KRAS/LKB1-mutant lung cancer cells. Nature. Published online May 24, 2017. doi:10.1038/nature22359 


\title{
A Practical Path Forward to More Patient-Centered Value Assessment
}

\author{
SACHIN KAMAL-BAHL, PHD; AND BRYAN LUCE, PHD, MBA
}

IN RECENT YEARS - and perhaps in reaction to rising health care costs and increased attention toward achieving greater efficiency out of current spending-various methodological frameworks have emerged that seek to assess the value of health care treatments and interventions and inform pricing, coverage, and reimbursement decisions.

As these critical endeavors move forward, one factor is emerging with growing consensus: the need for value assessment research to be more inclusive of the patient perspective. And while great strides have been made to determine what outcomes truly matter to patients and therefore should be foundational to assessing and tracking value in health care, the field is lacking a means to align value with patient-centered outcomes (PCOs) that can be captured, measured, and operationalized

Traditional value assessment methods, which rely on the conventional Quality Adjusted Life Year (QALY), have their limitations. In particular, basing a therapy's value solely on the QALY may overlook some elements of value that matter most to patients, such as the treatment's impact on their ability to work or stay active, the potential for reduced adverse effects, or the impact on family and caregivers.

These patient-derived value elements, such as the high caregiver burden experienced by family members and friends of patients diagnosed with complex diseases such as cystic fibrosis or multiple myeloma, the value of hope for treatment, and fear of contagion are often quantitatively excluded from traditional value assessment models. ${ }^{1}$ However, these costs can be substantial. A new study estimating the economic effects of coronavirus disease 2019 (COVID-19) found that China lost $\$ 275$ billion during the Chinese Lunar New Year week due to the fear of contagion. ${ }^{2}$ Ensuring such elements become a crucial part of the value conversation should be the end goal of any attempt to redefine measurements of treatment value.

But in order to ensure that value assessment accounts for what matters most to patients, we need a better understanding of the outcomes that are most important to patients. To date, some progress has been made on developing PCO measures, most of which have the goal of advancing research or clinical care.

Capturing the patient voice in value assessment has been an ongoing objective of the PhRMA Foundation's Value Assessment Initiative. ${ }^{3}$ Investigators at the Patient-Driven Values in Healthcare Evaluation (PAVE) Center ${ }^{4}$ at the University of Maryland are working to develop a framework for identifying patient-driven value elements for chronic conditions, starting with chronic obstructive pulmonary disease (COPD), childhood allergies, and hepatitis $\mathrm{C}$ virus.

A team at the University of Colorado's Center for Pharmaceutical Value (PValue) ${ }^{5}$ is testing methods to allow greater flexibility in accounting for attributes most important to stakeholders in assessing value in health care. Others, including the Center for Enhanced Value Assessment (CEVA) at Tufts Medical Center ${ }^{6}$ also aim to incorporate broader elements of value into economic evaluations.

So where do we go from here?

First, we need to identify all pertinent PCOs by disease, and perhaps even by severity within a specific disease state. We can learn from a number of ongoing efforts that aim to identify specific core outcome sets by disease. The Core Outcome Measures in Effectiveness Trials (COMET) initiative, ${ }^{7}$ which seeks to establish comprehensive reviews and a searchable database of core outcomes sets, offers a useful model. Other efforts such as the ICHOM standard sets (International Consortium for Health Outcomes Measurement), ${ }^{8}$ and the Green Park Collaborative's Core Outcomes Set Initiative ${ }^{9}$ are instructive as well.

These projects aim to establish a minimum set of core outcomes to be measured and reported in clinical studies for treatment of specific disease conditions and offer a useful model for how to incorporate patient perspectives into clinical research. However, it is not clear whether these efforts are sufficient to capture all pertinent PCOs, nor do these efforts appear to specifically link these core outcomes to treatment value.

We need to determine the degree to which existing activities are capturing pertinent PCOs and fill in the gaps where they may occur. Second, we must explore the effort to link all pertinent PCOs in a national repository that could serve as a go-to resource for anyone interested in understanding the value of health care interventions. This repository, which would organize key PCOs on a diseasespecific basis, could serve as a "one-stop-shop" for researchers looking to build patient-centered comparative effectiveness research or include patient-centered value elements into economic models. Such a repository could also inform outcomes-based contracting, quality measurement, and regulatory decision-making.

Establishing a PCO repository will require the active support of all stakeholders, including the patient community, payers, health outcomes researchers, and providers with disease-specific expertise to make it part of their mission. Redefining current value assessment methods in a manner that truly captures the patient perspective is a challenge for the entire health care community to tackle.

We have several steps to take before we can achieve such an endeavor, but working together to establish a common repository of PCOs will bring us closer to a health care system that offers the value all stakeholders are seeking.

\section{AUTHOR INFORMATION}

Sachin Kamal-Bahl, PhD, is president of COVIA Health Solutions. Bryan Luce, PhD, MBA, is an affiliate professor at the University of Washington School of Pharmacy.

\section{REFERENCES}

1. Lakdawalla DN, Doshi JA, Garrison LP, Phelps CE, Basu A, Danzon PM. Defining elements of value in health care-a health economics approach: an ISPOR special task force report. Value Health. 2018;21(2):131-139. doi:10.1016/j.jval.2017.12.007

2. Ma S, Kim DD, Cohen JT, Neumann PJ. Measuring "fearonomics effects" in valuing therapies: an application to Covid-19 in China. Value Health. 2020;23(11):1405-1408. doi:10.1016/j. jval.2020.06.002

3. Value assessment initiative. PhRMA Foundation. Accessed January 14, 2021. http://www. phrmafoundation.org/about-the-phrma-foundations-value-assessment-initiative/

4. Patient Driven Values in Healthcare Evaluation (PAVE) . University of Maryland School of Pharmacy. Accessed January 14, 2021. https:/ /www.pharmacy.umaryland.edu/centers/ patient-driven-values-healthcare-evaluation-pave/

5. Center for Pharmaceutical Value (PValue). Skaggs School of Pharmacy and Pharmaceutical Sciences. Accessed January 14, 2021. https://pharmacy.cuanschutz.edu/research/ research-centers/pvalue

6. New Center for Enhanced Value Assessment (CEVA) established at Tufts Medical Center. News release. Tufts Medical Center. February 1, 2019. Accessed January 14, 2021. https://cevr. tuftsmedicalcenter.org/news/2019/ceva COMET Initiative. Accessed January 14, 2021. https://www.comet-initiative.org/ Standard sets. ICHOM. Accessed January 14, 2021. https://www.ichom.org/standard-sets/ Green Park Collaborative. Accessed January 14, 2021. http:/ /www.cmtpnet.org/ green-park-collaborative/

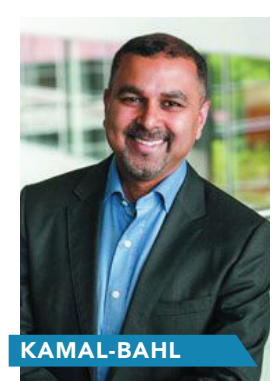

Sachin Kamal-Bahl, PhD president and founder of COVIA Health Solutions

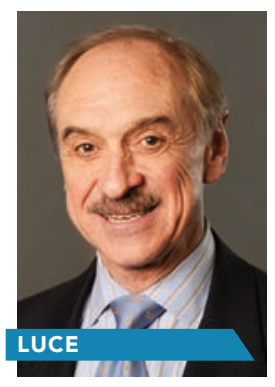

Bryan Luce, PhD, MBA, affiliate professor at the University of Washington School of Pharmacy 


\section{Azacitidine Is Shown to Improve Overall, Relapse-Free Survival in Older Adults With AML}

OLDER ADULTS WITH ACUTE myeloid leukemia (AML) saw increased overall survival (OS) and relapse-free survival (RFS), as well as preserved quality of life (QOL), following oral administration of azacitidine as maintenance therapy, according to study data published in the New England Journal of Medicine. ${ }^{1}$

The formulation of azacitidine used in the phase 3, randomized, double-blind, placebo-controlled QUAZAR AML-001 study was CC-486 (Onureg; Bristol Myers Squibb [BMS]), "a hypomethylating agent that is not bioequivalent to injectable azacitidine," the authors noted. As a result, CC-486 is not interchangeable with the intravenous and subcutaneous forms of azacitidine, BMS officials said in a statement announcing the FDA approval in September. ${ }^{2}$

In the study, with its primary end point of OS and secondary end points of RFS and health-related QOL, a 300-mg dose of CC-486 was administered each day for 14 days of a 28-day cycle. The 472 participants were randomized 1:1 to receive CC-486 $(\mathrm{n}=238)$ or placebo $(\mathrm{n}=234)$ between May 2013 and October 2017 , and the data cutoff point was July 15, 2019. CC-486 was given for a median of 12 (range, 1-80) cycles and placebo, 6 (range, 1-73) cycles.

Results show significant improvement in both OS and RFS with the use of CC- 486 . There was a $40 \%$ increase of OS compared with placebo; the CC- 486 and placebo groups saw a median OS from randomization of 24.7 vs 14.8 months $(P<.0001)$, respectively. For RFS, there was a 53\% difference: 10.2 vs 4.8 months $(P<.0001)$. Median follow-up was 41.2 months.

The trial was conducted as 148 sites in 23 countries. The median patient age was 68 (range, 55-86) years. Eligibility criteria included the following:

- Being 55 years or older

- Having newly diagnosed de novo AML (91\%) or secondary AML and intermediate- or poor-risk cytogenetic characteristics (86\%) at diagnosis

- Not being eligible for hematopoietic stem-cell transplantation

- Being in complete remission with/without complete blood count recovery

- Having a screening Eastern Cooperative Oncology Group score of 0 to 3

Before trial enrollment, all patients also received induction, while $78 \%$ of the CC- 486 group and $82 \%$ of the placebo group received at least 1 course of consolidation chemotherapy. The median time from induction therapy to randomization was 4.0 months; for complete remission to randomization it was 85.0 days.

Additional findings show an ongoing survival advantage with CC-486 vs placebo at the 1- and 2-year marks for OS. At 1 year, approximately $72.8 \%$ and $55.8 \%$ (difference, 17 percentage points; $95 \% \mathrm{CI}$, 8.4-25.6), respectively, were alive, whereas at 2 years, $50.6 \%$ and $37.1 \%$ (difference, 13.5 percentage points; 95\% CI, 4.5-22.5) were.

"An overall survival benefit was observed with CC-486 regardless of whether patients had received any consolidation therapy, had been in complete remission after induction chemotherapy, or had had persistent measurable residual disease at randomization," the authors noted.

Meanwhile, for RFS at 6 months and 1 year, the CC-486 and placebo groups came in at $67.4 \%$ and $45.2 \%$ (difference, 22.2 percentage points; $95 \%$ CI, $13.2-31.2$ ) and $44.9 \%$ and $27.4 \%$ (difference, 17.5 percentage points; $95 \% \mathrm{CI}$, 8.5-26.4), respectively.

For health-related QOL, no clinically meaningful differences were seen via least squares mean changes from baseline at any visit.

Dosing interruptions were seen in $43 \%$ of the CC- 486 and $17 \%$ of the placebo groups; dose reductions in $16 \%$ and $3 \%$, respectively; and discontinuation, $13 \%$ and $4 \%$.

The most common adverse events in both groups were grade $1 / 2$ gastrointestinal events (eg, nausea, vomiting, diarrhea) and grade $3 / 4$ thrombocytopenia and neutropenia.
Possible future areas of study are the molecular characteristics that predispose patients with AML to better outcomes following maintenance therapy and if CC-486 confers a survival advantage in other clinical contexts. •

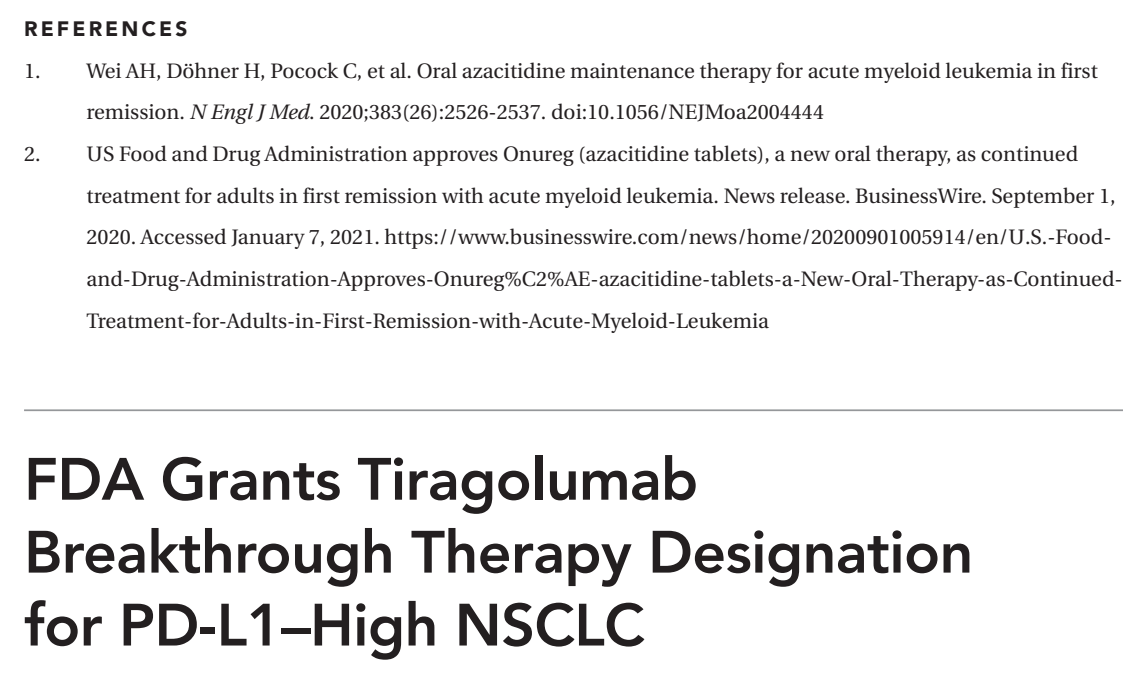

\section{FDA Grants Tiragolumab Breakthrough Therapy Designation for PD-L1-High NSCLC}

GENENTECH ANNOUNCED THAT TIRAGOLUMAB, a novel anti-TIGIT immunotherapy, was granted breakthrough therapy designation (BTD) by the FDA for use in combination with atezolizumab (Tecentriq) in the first-line treatment of metastatic non-small cell lung cancer (NSCLC) patients whose tumors have high levels of programmed-death ligand-1 (PD-L1) and do not have EGFR or anaplastic lymphoma kinase $(A L K)$ mutations. ${ }^{1}$

Tiragolumab is the first anti-TIGIT therapy to be granted BTD by the FDA The designation was based on data from the phase 2 CITYSCAPE trial that was first presented at the 2020 American Society of Clinical Oncology (ASCO) Virtual Scientific Program in May. TIGIT is an immune checkpoint protein that is expressed on multiple immune cells, including T cells.

The double-blind, placebo-controlled trial included 135 chemotherapy-naïve, PD-L1-positive (tumor proportion score [TPS] $\geq 1 \%$ determined by 22C3 IHC pharmDx Dako assay) NSCLC patients with locally advanced or metastatic disease and no EGFR or ALKmutations. ${ }^{2}$ Primary end points are overall response rate (ORR) and median progression-free survival (PFS).

Patients were randomized to receive either tiragolumab plus atezolizumab $(n=67)$ or atezolizumab plus a placebo $(n=68)$, and an initial analysis with median follow-up of 5.9 months found that the tiragolumab cohort had a better ORR and longer median PFS compared with the placebo group. Treatment-related adverse events (TRAEs) occurred in $72 \%$ of the placebo group and $80.6 \%$ of the tiragolumab cohort. The full results presented at ASCO provided an additional 6 months of follow-up, with a median follow-up of 10.9 months as of December 2, 2020.

ORR and median PFS remained improved in the tiragolumab cohort compared with the placebo group. Tiragolumab-treated patients saw a 37.3\% ORR and 5.6-month median PFS versus $20.6 \%$ and 3.9 months in patients on the atezolizumab and placebo regimen. In an exploratory analysis, people whose tumors expressed high levels of PD-L1 (TPS $\geq 50 \%$ ) showed a $66 \%$ ORR in the combination therapy group versus $24 \%$ with atezolizumab alone. The safety profile remained tolerable with the combination therapy.

The CITYSCAPE trial is the first randomized trial of an anti-TIGIT therapy and provides evidence that targeting both TIGIT and PD-L1 may improve anti-tumor activity by amplifying immune response. Biomarker analyses from the trial will be presented at the International Association for the Study of Lung Cancer 2020 World Conference, which is set for January 28-31, 2021.

"We have been researching TIGIT as a novel cancer immunotherapy target for almost 10 years and we are pleased that the FDA has acknowledged the potential of tiragolumab to substantially improve outcomes for people with certain types of lung cancer," Levi Garraway, MD, PhD, chief medical officer and head of global product development at Genentech, said in a statement. "We look forward to advancing our tiragolumab development program, which 
includes chemotherapy-free combinations and trials in early stages of disease across multiple cancer types with high unmet need."

The tiragolumab development program aims to expand into earlier disease stages and new patient populations with unmet needs. There are ongoing randomized trials in metastatic NSCLC and small cell lung cancer; earlier disease stages such as stage III NSCLS and locally advanced esophageal cancer; and metastatic esophageal squamous cancer and cervical cancer. •

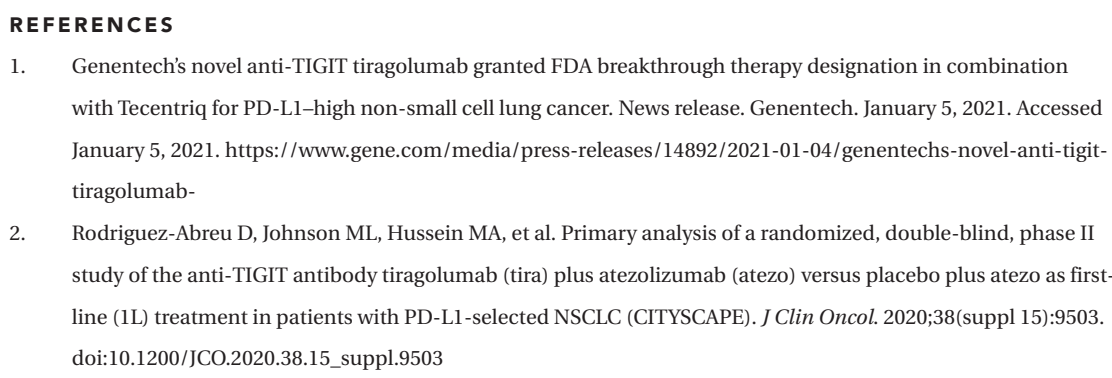

\section{FDA Updates Ibrutinib Label With 5-Year Data in Waldenström Macroglobulinemia}

THE FDA UPDATED PRESCRIBING INFORMATION for ibrutinib (Imbruvica), a Bruton tyrosine kinase (BTK) inhibitor approved to treat Waldenström macroglobulinemia (WM), to include 5 years of efficacy data, AbbVie announced in a press release.

The approval of updated prescribing information is based on positive safety and efficacy data from the final analysis of the phase 3 iNNOVATE study, which were presented at the 2020 American Society of Hematology (ASH) meeting. At an overall follow-up of 5 years, combination ibrutinib and rituximab therapy led to significantly longer progression-free survival (PFS) and was more effective than rituximab monotherapy in both previously treated and treatment-naïve patients. ${ }^{2}$

The iNNOVATE trial randomized 150 patients to receive either ibrutinib and rituximab or rituximab monotherapy. Patients in the ibrutinib arm of the trial had a $75 \%$ lower risk of disease progression and death than those in the rituximab monotherapy group (HR, 0.25 ; 95\% CI, 0.15-0.42; $P<.0001$ ).

"We're encouraged by this latest recognition from the FDA as it underscores our commitment to supporting those impacted by Waldenström's macroglobulinemia, a rare and incurable form of non-Hodgkin lymphoma," Danelle James, MD, MAS, Imbruvica global development lead, said in a statement. "Imbruvica is the only FDA-approved treatment for these patients and now includes more than 5 years of safety and efficacy data to help provide better understanding of how to treat this rare blood cancer."

Ibrutinib is currently approved for patients with several types of blood cancer both as a monotherapy and in combination with rituximab, and the data presented at ASH are currently the longest available for any BTK inhibitor in WM.

"The long-term results from the phase 3 iNNOVATE study provide clinicians even more evidence that patients with WM can benefit from treatment with an ibrutinib-based regimen and maintain prolonged progression-free survival," said principal investigator Meletios A. Dimopoulos, MD, professor and chairman of the Department of Clinical Therapeutics at the National and Kapodistrian University of Athens School of Medicine in Athens, Greece. •

\section{REFERENCES}

1. Imbruvica (ibrutinib) US prescribing information updated to include long-term data for Waldenström'

macroglobulinemia (WM). News release. AbbVie. December 23, 2020. Accessed December 23, 2020. https:// www.prnewswire.com/news-releases/imbruvica-ibrutinib-us-prescribing-information-updated-to-in-

clude-long-term-data-for-waldenstroms-macroglobulinemia-wm-301197891.html
2. Buske C, Tedeschi A, Trotman J, et al. Five-year follow-up of ibrutinib plus rituximab vs placebo plus rituxima for Waldenstrom's macroglobulinemia: final analysis from the randomized phase 3 iNNOVATE study. Paper presented at: American Society of Hematology Annual Meeting; December 5-8, 2020. Abstract 336. https://ash. confex.com/ash/2020/webprogram/Paper134460.html

\section{As Mammography Screenings Drop, Advanced Breast Cancer Cases Increase in First Months of COVID-19}

IN THE FIRST FEW MONTHS of the coronavirus disease 2019 (COVID-19) pandemic, mammography screenings dropped drastically and patients with breast cancer presented with more advanced and aggressive disease, according to study findings presented at the 2020 San Antonio Breast Cancer Symposium.

The retrospective, double-cohort study observed patients visiting the Kaiser Permanente health care system, comparing those who visited the health system between March 17 and May 18, 2020_during California's shelter-in-place period - to those who visited the health system between March and May 2019.

"The COVID-19 pandemic drove operational changes in Kaiser Permanente California," said Annie Tang, MD, researcher at Kaiser Permanente Northern California's Permanente Medical Group Breast Research Collaborative. She said that on March 17, 2020, when the California shelter-in-place order was initiated, "Breast cancer guidelines were developed within our large integrated health care system of 22 hospitals, all outreach for mammography screenings were held, but women who wished to have screening were still accommodated, elective surgeries were stopped, and cancer surgeries were continued."

There were 703 patients with newly diagnosed disease identified in the 2019 period, compared with 250 during the shelter-in-place period, representing a $64 \%$ drop in the number of patients who received a diagnosis and potentially highlighting that there were 453 patients who had undiagnosed disease during this time, Tang said.

Both groups of patients had similar baseline characteristics, although there were significantly more patients in the 2020 period who presented with symptomatic disease (78\% vs $37 \%$ ) and presented with more advanced disease, as measured by $>$ T1c tumors $(78 \%$ vs $64 \%$; $P<.001)$, positive nodal status $(24 \%$ vs $18 \% ; P=.07)$, and metastatic disease ( $7 \%$ vs $2 \% ; P=.002)$.

Patients in 2020 also had tumors of more aggressive receptor subtypes. More patients in 2020 had hormone receptor-positive (HR+)/human epidermal growth factor receptor 2-positive (HER2+) disease (13\% vs 9\%) and HR-negative (HR-)/HER2-negative (HER2-) disease (16\% vs 10\%), while they had less HR+/HER2- disease (66\% vs 76\%) and less HR-/HER2+ disease (4\% vs 5\%).

"As screening mammography rates return to prepandemic levels, our system must prepare for a likely surge of new breast cancer diagnoses," Tang said. "We will continue to monitor breast cancer screening rates, breast cancer diagnosis, and treatment trends for future research."

Although the majority of initial treatment was surgery for both 2020 and 2019 , the percentage of patients getting surgery decreased in 2020 (71\% vs $83 \%$ ) as the percentage of patients getting chemotherapy as initial treatment increased (15\% vs $10 \%)$. More patients also underwent neoadjuvant hormone therapy in 2020 (5\% vs 0\%).

Notably, the time to first treatment was shorter in 2020, with time to surgery dropping from 26 to 19 days and time to chemotherapy dropping from 28 to 24 days.

\section{REFERENCE}

1. Chang S, Savitz A, Vuong B, et al. Characterization of breast cancer management during the COVID 19 pandemic in a large integrated healthcare delivery system: Stage at diagnosis and timing/modality of first treatment. Presented at: San Antonio Breast Cancer Symposium; December 8-11, 2020. Abstract SS2-06. https://www. abstractsonline.com/pp8/\#!/9223/presentation/704 


\section{Lung Cancer Screenings Fall, Biopsy Rates Rise During COVID-19}

LUNG CANCER SCREENINGS are critical for early detection and timely treatment, but the coronavirus disease 2019 (COVID-19) pandemic has made keeping up with routine care, including screenings, more difficult. A study published as an article in press by the Journal of the American College of Surgeons found that deferred care in the spring of 2020 as the COVID-19 pandemic first surged has worsened outcomes for patients with lung cancer. ${ }^{1}$

LDCT screening reduces lung cancer mortality by at least $20 \%$ in high-risk patients, and the current United States Preventive Services Task Force guidelines recommend low-dose computed tomography (LDCT) screening for adults aged 55 to 80 years, a 30 pack-year smoking history, and currently smoking or those who quit within the last 15 years.

Researchers from the University of Cincinnati aimed to gauge the impact of deferred LDCT due to the unprecedented shutdown caused by the pandemic. They suspended screenings on March 13 and started a phased reopening on May 5, fully opening on June 1 . They compared the monthly screening visits during March 2020 to July 2020 to those in the 3 years prior (January 2017-February 2020).

"We had 800 scans cancelled during that time and even during the resumed period, we had a decreased total volume of patients scanned and also noted a decreased number of new patients who were scanned for their lung cancer screening," lead author Robert M. Van Haren, MD, MSPH, FACS, assistant professor and thoracic surgeon at the University of Cincinnati College of Medicine, said in a statement. ${ }^{2}$

In all, 800 screening visits were canceled in that time frame, with total monthly visits dropping from an average of 146 to an average of 39. There were just 17 new patient visits for LDCT screening monthly on average during the pandemic, versus 56 at baseline. Even once the institution was back at full operations, the rates remained reduced.

"When we resumed our operations, we found that new patients were less likely to come back to our screening program," Van Haren said. “...We also found that patients were more likely not to show up for their CT appointments, and this rate was again significantly increased compared with baseline."

The no-show rate rose from $15 \%$ before the pandemic to $40 \%$ after restrictions were implemented.

Once the clinic reopened, the percentage of patients who had potentially malignant nodules on their lungs after screening was higher $(8 \%$ vs $29 \%$, respectively). Therefore, more patients were referred to specialists for either biopsies or surgery during that time.

"Our results are important and suggest that it's critical to continue cancer screening operations, such as our lung cancer screening, during this pandemic," Van Haren said. "It's maybe more important now as we continue to undergo another surge of COVID-19 cases throughout the country."

To ensure screening is safe, even during the pandemic, the site of care was changed from an in-hospital setting to an outpatient center, social distancing protocol was implemented in waiting areas and scanning rooms, and appointments were spaced out further to allow for cleaning between patients. Clinicians also put an emphasis on educating patients to reinforce the message that it is safe to go for screenings during this time, Van Haren said.

"We have to make sure as health care providers that we're taking care of patients who have COVID-19 and along with the rest of the population who don't have COVID-19," Van Haren said, "and that those 2 patient populations can safely co-exist and receive treatment." •

\section{REFERENCES}

1. Van Haren RM, Delman AM, Turner KM. Impact of the COVID-19 pandemic on lung cancer screening program and subsequent lung cancer. J Am Coll Surg. Published online December 17, 2020. doi:10.1016/j.jamcollsurg.2020.12.002

2. Study reports drop in lung cancer screening, rise in malignancy rates during spring COVID-19 surge. News release. American College of Surgeons. December 17, 2020. Accessed December 18, 2020. https://www.newswise.com/ articles/study-reports-drop-in-lung-cancer-screening-rise-in-malignancy-rates-during-spring-covid-19-surge

\section{Proximity to Treatment, Racial Disparities Are Seen in Ovarian Cancer Survival}

PATIENTS WITH OVARIAN CANCER who lived within 10 miles of Kansas's only National Cancer Institute (NCI)-designated cancer center were shown in a recent study to have less of a survival advantage because of their location, according to the results published in The American Journal of Surgery.

Using a cutoff distance of 10 miles, the investigators found that patients with ovarian cancer who lived within that geographic area had worse overall survival, which was the study's primary outcome. The effects of 3 distances were evaluated among 220 women (mean [SD] age, 60.6 [11.0] years) who received care at the University of Kansas Cancer Center (KUCC) between 2010 and 2015:

- Up to 10 miles $(\mathrm{n}=49)$

- $\quad 11$ to 50 miles $(\mathrm{n}=128)$

- $\quad$ More than 50 miles $(n=43)$

Although racial disparities in survival are well documented in the ovarian cancer space, the impact on receipt and quality of treatment is not.

"The primary objective of this study was to determine if geographic distance from a single NCI-CC in Kansas (with a large catchment area that spans the entire state of Kansas and western Missouri) is associated with differences in survival," the authors noted. "We predicted that like prior studies, distance traveled would be negatively associated with receipt of guideline adherent care and survival."

International Classification of Diseases, Ninth Revision and 10th Revision codes confirmed a diagnosis of ovarian, fallopian tube, and primary peritoneal cancer. Patients were excluded if their chemotherapy or surgical staging was provided by another institution. Most (88\%) of the patients were White.

The results show that more non-White patients $(P=.0020)$ lived no more than 10 miles from the cancer center. In addition, at diagnosis, most patients had papillary serous histology (75\%) and stage III disease (60\%). The similarities seem to end there.

Optimal cytoreductive surgery was more likely to occur among the patients who lived more than 10 miles away compared with those who lived up to 10 miles away: $83 \%$ vs $65 \%(P=.0276)$. This despite $84 \%$ of patients overall undergoing the surgery. Patients who lived closer also had an increased mortality risk (HR, 1.61; 95\% CI, 1.07-2.42; $P=.0229$ ) and a lower 5-year survival rate compared with those who lived farther: $36 \%$ vs $55 \%$.

No difference in progression-free survival was seen when the distance was considered.

There was a surgical gap seen, too, when stratifying by race. Lower rates of optimal cytoreduction were seen among the non-White (Black, Asian, Latina, and "other") patients vs the White patients and overall: $67 \%$ vs $81 \%$ vs $80 \%$, respectively. These patients were also less likely to receive National Comprehensive Cancer Network guideline-adherent care: $78 \%$ vs $87 \%$ vs $85 \%$.

"After controlling for age at diagnosis, non-White race, late stage at diagnosis, presence of comorbidities, median income, and suboptimal cytoreduction, geographic distance alone is not an independent predictor of worse overall survival," the authors concluded. "[However], it could serve as a proxy for poor social determinants of health and accompanying aggressive tumor biology among patients."

This is because fewer debulking surgeries happened among patients who lived within 10 miles of KUCC and more of these patients did not undergo surgery at all.

\section{REFERENCE}

1. Petersen S, Shahiri P, Jewell A, et al. Disparities in ovarian cancer survival at the only NCI-designated cancer center in Kansas. Am J Surg. Published online December 7, 2020. doi:10.1016/j.amjsurg.2020.12.009 


\begin{abstract}
AJMC ${ }^{\circledR} T V$ interviews let you catch up on what's new and important about changes in health care, with insights from key decision makers-from the clinician, to the health plan leader, to the regulator. When every minute in your day matters, AJMC ${ }^{\circledR}$ TV interviews keep you informed. Access the video clips at ajmc.com/interviews.
\end{abstract}

\section{Bradley Monk, MD, FACOG, FACS on PARP Inhibitors in Ovarian Cancer Treatment}

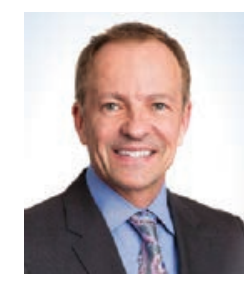

\author{
MONK IS A CLINICIAN AT ARIZONA ONCOLOGY, \\ PROFESSOR AT THE UNIVERSITY OF ARIZONA \\ AND CREIGHTON UNIVERSITY IN PHOENIX, HEAD \\ OF THE US ONCOLOGY GYNECOLOGIC RESEARCH \\ COMMITTEE, AND CURRENT CO-DIRECTOR \\ FOR THE GYNECOLOGIC ONCOLOGY GROUP \\ RESEARCH CONSORTIUM.
}

Can you discuss the current approved indications for PARP inhibitors in ovarian cancer?

Poly (ADP-ribose) polymerase, or PARP, inhibitors are oral anti-cancer therapies. They're approved in 4 tumor types-breast, there are 2 of them; pancreatic; and then in prostate, there are also 2 of them. And in those breast, pancreatic and prostate, there are molecularly defined populations. I'd like to talk to you about the ovarian cancer indications.

We have 9 PARP inhibitor indications in ovarian cancer. I can kind of put them into groups. The first PARP approved in treatment, that first group, was called olaparib, in December 2014. So we've been using PARP inhibitors in the clinic for 6 years. And olaparib was an accelerated approval, as were the others: rucaparib, and also niraparib; again, in the treatment setting of later lines of therapy. They're all molecularly defined populations. Olaparib is germline $B R C A$ mutation, rucaparib is germline and somatic mutation, and niraparib is $B R C A$ mutation or homologous recombination repair-deficient, platinum-sensitive cancer; so patients who have a platinum-sensitive relapse that have the molecular signature, which we abbreviate HRD.

How has the reimbursement landscape for PARP in inhibitors evolved since the first one was approved? Do patients have any access issues that are driven by reimbursement decisions?

Reimbursement for PARP inhibition has been universal in the United States and even in Europe. These new frontline PARP inhibitor indications of PRIMA [trial] and the PAOLA-1 [trial] —PRIMA niraparib alone, PAOLA-1 bevacizumab, and olaparib-have now been approved in Europe. Europe is trafficking through reimbursement, but in the United States they are universally paid for. The only caveat is that in the bevacizumab-olaparib combination, there is a companion diagnostic, so generally reimbursement is only for those patients who have the molecular HRD signature.

Even though PARP inhibitors have been paid for through insurances, which is universally true, there are still co-pays, and co-pays are the real financial burden. The companies try to support through copay assistance programsthey have foundations - but there still is a substantial financial burden to these expensive oral medications. Someday there'll be generic, and we already have multiple biosimilars for bevacizumab, and we're seeing the prices come down.

So, in the end, it's all about value. And the value of PARP inhibitors, obviously, is the most in patients with a BRCA mutation-either germline or somatic, it's about the same- -but also a lot of value in the HRD, BRCA-like gene subset.
The value in the patients that are what we call homologous recombination proficient is not as much, but they're still approved, and certainly there is a value issue with bevacizumab. And when you add bevacizumab and olaparib together, then obviously 2 medications are more expensive than 1 .

In your experience, who are the patients who fare best with PARP inhibitors for ovarian cancer?

The first randomized trials to show efficacy of PARP inhibitors were these 3 maintenance treatments in recurrent ovarian cancer, and olaparib has 2 studies, Study 19 and SOLO2; niraparib has one, NOVA; and then rucaparib has 1, ARIEL3. You can now use PARP inhibitors in treatment in recurrent ovarian cancer based on level I randomized trial data, if the patient responds to platinum, across all biomarker subgroups, because response to platinum in the recurrent setting is the biomarker. So again, if you treat a patient in platinum-sensitive relapse with carboplatin-paclitaxel, or carboplatin-liposomal doxorubicin, or even carboplatin-gemcitabine, if she responds, you can keep her in response with either olaparib, rucaparib, or niraparib without sort of ruining the patient experience. All of those studies have no decrement in patient reported outcomes, and no new safety signals identified.

"The biggest issue here is patients not being tested for biomarkers. So before we talk about what knowledge gaps there are in genomic testing [in prostate cancer], I think we need to test all patients first, and then talk about the gaps."

—Neeraj Agarwal, MD, professor of medicine, physician, and director of the Genitourinary Oncology Program at the University of Utah's Huntsman Cancer Institute

What have been some key developments in the regulatory landscape of PARP inhibitors since they were first approved in ovarian cancer in 2014?

The most recent advancement in PARP inhibitors has been the use of PARP inhibitors in frontline, newly diagnosed advanced ovarian cancer. The first really amazing study was SOLO1, published in the New England Journal of Medicine. ${ }^{1}$ The first author is Kathleen Moore. And that showed in maintenance frontline therapy-again, now in the molecularly defined population of BRCA only-had a dramatic improvement in progression-free survival. And we're hoping that someday, frontline, PARP inhibition in newly diagnosed advanced ovarian cancer in BRCA patients will improve overall survival.

SOLO1 was a practice-changing, licensing-enabling, reimbursement sort of definitive trial, but only in patients with BRCA-mutated, newly diagnosed advanced ovarian cancer that responded to chemotherapy. The challenge, though, was it was in a very well-defined but small patient population, those " 
that had somatic or germline BRCA mutations. So somatic mutations, tumor tests, maybe $5 \%$ to $10 \%$; in germline, maybe $15 \%$ to $20 \%$. So, it was only about a quarter of the patients. So then what happened is we launched this international collaboration to go beyond patients with a BRCA mutation. And there is a molecular signature that I've sort of alluded to, homologous recombination repair deficiency [HRD], which can be ordered either through Foundation Medicine or through Myriad, which identifies BRCA-like genes where there might be a broader opportunity for PARP inhibition.

We launched that study collaborating with the GOG [Gynecologic Oncology Group] in the European Network, and we showed that we could utilize PARP inhibitors beyond $B R C A$, this time, niraparib in a study called PRIMA published in the New England Journal of Medicine in December of 2019. ${ }^{2}$ [It was] FDA approved on April 29, 2020, beyond BRCA. In fact, it works so well that the FDA said not only can you use it in BRCA, but BRCA-like genes, and HRD. You can use it in all comers. And the study personalized the dose, and niraparib is the only once-daily PARP inhibitor, so a very distinguishing quality. Beyond $B R C A$, all comers, once daily, and a personalized dose.

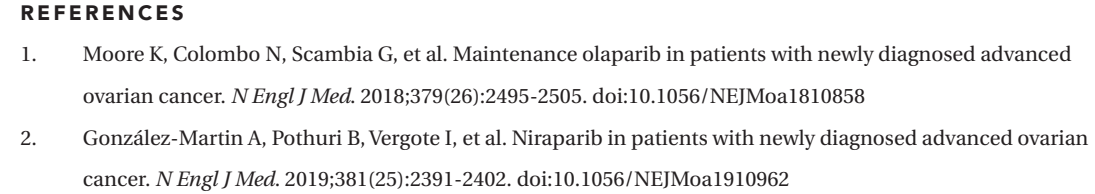

\section{Neeraj Agarwal, MD, Discusses PARP Inhibition in Prostate Cancer}

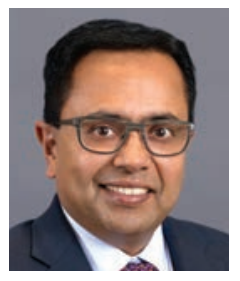

AGARWAL IS A PROFESSOR OF MEDICINE, PHYSICIAN, AND DIRECTOR OF THE GENITOURINARY ONCOLOGY PROGRAM AT THE UNIVERSITY OF UTAH'S HUNTSMAN CANCER INSTITUTE.

In your opinion, who is an appropriate candidate for a PARP inhibitor in prostate cancer?

Everyone who has a DNA repair gene defect, or homologous recombination repair defect, is a candidate for treatment with a PARP inhibitor. If we don't identify these patients, we are offering them the alternate treatment, which is chemotherapy. And we know from multiple data sets, larger real world data sets such as flat RM, for example, the real-world use of chemotherapy is very low in patients with metastatic castrate-resistant prostate cancer, which basically tells us that our patients in the real world are not really eligible or enthusiastic about receiving chemotherapy in castrate-resistant prostate cancer. How to make them eligible and to maximize eligibility for treatment with PARP inhibitors is the most important question, which can only be answered by testing all our patients to look for these mutations.

What knowledge gaps remain in genomic testing and biomarker development in prostate cancer?

The biggest issue here is patients not being tested for biomarkers. So before we talk about what knowledge gaps there are in genomic testing, I think we need to test all patients first, and then talk about the gaps. As we know, olaparib and rucaparib are 2 PARP inhibitors currently approved for patients with advanced prostate cancer. Olaparib is approved for patients who are progressing on novel hormonal therapies, and rucaparib was approved for patients who are progressing on novel hormonal therapies and chemotherapy. So, any patient with those eligible mutations is eligible for treatment with PARP inhibitors. But how many patients are actually being tested for those mutations?

The answer is, we do not know yet, because the drugs just got approved. But based on the data, we have, less than half of the patients are being tested. In fact, in the PROfound trial, which led to approval of olaparib, $30 \%$ of patients were not even eligible to screen for the trial because tissue was not available for testing. I think the biggest gap here is we need to test these patients the first time we see them in the clinic. When we order CT scans, we order bone scans, and order laboratory data, I think we should order comprehensive genomic profiling of the tumor. And depending upon the resources, refer to the high-risk genetics clinic or germline testing at the same time for all patients with prostate cancer. I think that's a first barrier I see before we identify more gaps.

And the second knowledge gaps are why patients do not respond in similar fashion. Why is response to PARP inhibitors in patients with prostate cancer with $B R C A 1$ mutation, for example, not as robust as we see in patients with ovarian cancer who have $B R C A 1$ mutation? Why for BRCA2 mutation, do we have differential responses for patients who have heterozygosity for $B R C A 1$ loss versus homozygosity for $B R C A 2$ loss? I think there are a lot of questions which can be answered. But the first step would be to test all these patients, so that we know at least what is going on in the majority of the patients. And not only to answer these scientific questions, but also to make these oral pills which are so much more tolerated than contemporary chemotherapy, which are the other options in the study. Testing the patients is the biggest gap we have, and we need to test everyone we see them on the first or second time.

There is discussion of giving PARP inhibitors earlier in the course of treatment. What are your thoughts on this issue?

As far as] moving PARP inhibitors from the late metastatic castrate-resistant prostate cancer setting to early on, there are 3 trials going on, which are testing PARP inhibitors in first-line metastatic CRPC setting. Right now, PARP inhibitors are approved for after patients have been treated with novel hormonal therapy in metastatic castrate-resistant prostate cancer, for olaparib and a novel hormonal therapy plus chemotherapy with docetaxel for rucaparib.

There are 3 trials which are going on including the TALAPRO-2 trial, MAGNITUDE trial, and the PROPEL trial. They are asking the question of whether PARP inhibitors may have a role in patients who are newly diagnosed with castrate-resistant prostate cancer. Another trial has started in metastatic castration-sensitive prostate cancer-so again, moving the PARP inhibitors to more upfront settings in newly diagnosed patients with metastatic castration-sensitive prostate cancer. A trial has recently started known as the AMPLITUDE trial. And in that trial, hundreds of patients who are candidates for this trial, meaning they have to have DNA repair, gene-related defects in the cancer cells, will be randomized to abiraterone plus androgen deprivation therapy versus abiraterone plus androgen deprivation therapy plus niraparib. This trial is just open to accrual in the metastatic castration-sensitive prostate cancer, and the only way to move these agents to upfront settings is to accrue on the trial. Awareness among the patients is very important, and the onus is on us now to recruit patients on these trials so that we can get results fast enough to make these drugs available for our patients early on.

PARP inhibitors are being studied in combination with other therapies-which regimens being studied do you believe hold the most promise in prostate cancer?

I think the combination of PARP inhibitors with novel hormonal therapy definitely has promise. Whether it's a combination of talazoparib with enzalutamide as being tested in TALAPRO-2 trial, or niraparib with abiraterone in the MAGNITUDE trial, and then olaparib with abiraterone in the PROPEL trial, I think all of these combinations have promise. Also, the AMPLITUDE trial, as I said early on, for patients with metastatic castrate-sensitive prostate cancer, is a combination of abiraterone with niraparib that has promise, too. As a class, PARP inhibitors with novel hormonal therapy have a lot of scientific rationale. There is another clinical trial going on, the KEYLYNK trial, where olaparib is being tested in combination with pembrolizumab, which is an immune checkpoint inhibitor, in late metastatic castrate-resistant prostate cancer. I think that combination is also promising. Ultimately, the data will tell us whether these combinations are going to be approved or not, within next 1, 2, or 3 years. So we're really looking forward to the results of those trials. 


\section{Radiation Oncology's Role in the Next Chapter of Payment Reform}

ANNE HUBBARD, MBA

CONTINUED FROM COVER

This seems like a relatively straightforward goal to achieve, but based on our experience spanning 2 , and now 3 , administrations, it is actually an incredible challenge. As willing participants in the march toward greater use of value-based payment initiatives, our efforts have been stymied by Medicare officials' desire to simply cut payments, rather than improve the overall care delivery system. This shortsighted approach must be replaced with a more collaborative and transparent approach that puts patients first.

\section{Nominal Risk, Not Financial Jeopardy}

The Medicare Access and CHIP Reauthorization Act of 2015 (MACRA) requires that a nominal amount of risk be established to meet Advanced APM status requirements. ${ }^{3}$ The American Society for Radiation Oncology (ASTRO) has always appreciated that risk is required under the APM construct to not only ensure physician investment in the management of the cost of care, but also to secure Medicare savings. However, the application of a $3.75 \%$ discount off the professional component (PC) and $4.75 \%$ discount off the technical component (TC) payments is a massive cut that fails to recognize that radiation oncology relies on advanced technology and equipment that require a significant financial investment, beyond anything else in medicine.

The minimum total capital required to open a freestanding radiation oncology center is approximately $\$ 5.5$ million. An additional minimum $\$ 2$ million is necessary for annual operating and personnel expenses. These significant fixed investments far outweigh the variable costs of operating a radiation oncology clinic and should be given much greater consideration as part of any APM. While it is important to reduce the cost of care and drive value in health care, it is also important to ensure that efforts to generate savings do not jeopardize access to care by limiting practices' ability to offer state-of-the-art radiation therapy delivered by expert clinical staff.

CMS is misguided in its aggressive approach to secure savings from radiation oncology, the most cost-effective cancer treatment for Medicare beneficiaries. Over the last 10 years, radiation oncology total allowable charges have represented a declining portion of the total allowable charges in the Medicare Physician Fee Schedule (MPFS). The overall \$47 million/3\% decline in allowable radiation oncology charges between 2010 and 2020 pales in comparison to the overall $\$ 15.7$ billion/17\% increase in total MPFS allowable charges over the same period.

Moving forward, APMs need to consider the financial context in which care is delivered by asking key questions to guide the application of nominal risk. This includes an analysis of variableto-fixed cost ratios, changes to existing allowable charges over time, as well as consideration of total operating and personnel costs. Otherwise, models will be subjected to one-size-fits-all risk requirements, which will put APM participants in financial jeopardy and create access-to-care issues.

Increase Transparency Through Broader Collaboration and Data Sharing

In 2015, following the passage of the MACRA, ASTRO successfully lobbied for passage of the Patient Access and Medicare Protection Act (PAMPA), which initiated CMMI's work on an APM for radiation oncology. In April 2017, after years of internal work and frequent collaboration with CMMI, ASTRO proposed an APM, from which many concepts in the CMS RO Model can be found. Once the proposed rule was issued in 2019, ASTRO provided CMS with constructive comments and recommendations, which were echoed by the radiation oncology community, broader health care stakeholders, cancer patients, and numerous bipartisan Congressional leaders. Given this history, we were shocked that most of our recommendations were not considered in the final rule. ${ }^{4}$

The RO Model proposed rule included a comprehensive Medicare beneficiary data file but no detail on the methodologies used to formulate case-mix or practice historical experience that would allow for replication. ASTRO appreciated the disclosure of the data files and encouraged CMMI to provide more detail on the case mix and historical experience methodologies in the final rule. However, CMMI disregarded this request and did not provide Medicare beneficiary data files in the final rule, despite changes in the payment methodology.

The agency issued a subsequent "corrections" document that provided a vague explanation regarding a significant shift in Medicare Fee-for-Service (FFS) revenues for those practices participating in the RO Model. Physician group practices were expected to experience a $6 \%$ decrease in FFS payments based on the final rule, which was modified to a $1.6 \%$ increase in the correction document. For hospital outpatient departments, the correction document increased the anticipated cuts from $4.7 \%$ to $8.7 \%$. The lack of transparency regarding how this data was calculated causes us great concern. Sharing this type of information allows others to replicate the methodology and determine whether there are issues that warrant further consideration. Otherwise, we will be unable to fully understand the impact of the model across the entire specialty, let alone the specific impact on practices that are compelled to participate.

The process associated with the development of APMs must involve greater transparency and meaningful collaboration with CMS and CMMI officials to secure more balanced models that provide higher quality and lower costs. This includes engagement not only with CMMI policy staff, but also with actuarial staff and others who are involved in APM development. Data sharing and detailed explanations regarding calculations and methodologies used to determine payment rates are critical. CMS has started pushing for more price transparency across the health care spectrum; the same standard should be applied to payment methodologies developed by the agency.

\section{Ensuring Access to Guideline Concordant Care and Addressing Health Care Disparities}

The RO Model does not recognize the importance of guideline concordant care and, in some cases, discourages its use. The model's national base rates for cervical cancer do not reflect the costs of care delivered with both external beam therapy and brachytherapy, which is the standard of care for women. ASTRO's analysis, based on the proposed rule data files, found that the average PC and TC allowed charges were $29 \%$ and $16 \%$ more than the national base rates.

These rates fail to recognize that a significant portion of cervical cancer cases involve multiple physicians: one radiation oncologist at one site delivering the external beam therapy and another radiation oncologist at another site delivering the brachytherapy. "

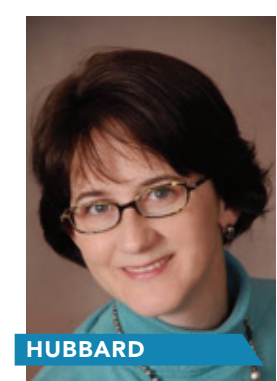

Anne Hubbard, MBA, director of health policy at the American Society for Radiation Oncology 
Treatment from multiple physicians allows some patients, particularly those in rural areas, to receive brachytherapy from high-volume, expert specialists, while still receiving external beam therapy more conveniently close to home, reducing travel time and costs.

Since cervical cancer is predominantly seen in women with poor access to health care, this decision further widens the health care disparities for socioeconomically disadvantaged populations. Payment methodologies must be developed based on guideline concordant care. They must also incorporate mechanisms to modify payment based on the wraparound services that vulnerable patient populations may need to successfully complete treatment. The existing RO Model discourages the use of guideline concordant care and does not address health care disparities.

\section{Mitigate Financial Toxicity for} Medicare Beneficiaries

Due to the RO Model's episode-based payment methodology, Medicare FFS beneficiaries will be required to pay $20 \%$ of the bundled payment amount that the practice or facility receives.
This means that patients who receive fewer or lower-cost services than average for their type of cancer would pay more in cost-sharing than if they had received the same treatment in a nonparticipating region, whereas patients who receive more services than average would pay less in cost-sharing.

Health care financial toxicity causes two-thirds of American bankruptcies. ${ }^{5}$ CMS must ensure that payment models do not exacerbate this problem. Models should base patient cost sharing on the lesser of (a) what the patient would have paid in cost-sharing under standard Medicare payment amounts for the specific services the patient received or (b) $20 \%$ of the bundled payment.

\section{The Path Forward}

While ASTRO continues to support the transition from FFS to value-based payment, we believe that CMMI's approach needs an overhaul. Through improved collaboration and transparency, we see a path forward and welcome the opportunity to discuss improvements to the RO Model with the Biden administration. While we can all agree that Medicare spending needs to be reined in, we must ensure that these efforts do not jeopardize patient care.

\footnotetext{
AUTHOR INFORMATION

Anne Hubbard, MBA, is director of Health Policy for the American Society for Radiation Oncolo

REFERENCES

1. Verma S. Correcting the course of value-based care. Modern Healthcare. Published October 27, 2020. Accessed January 13, 2021. https:/ /www. pcpcc.org/2020/10/27/correcting-course-value-based-care-models

2. Navathe AS, Emanuel EJ, Glied S, Mostashari F, Kocher B. Medicare payment reform's next decade: a strategic plan for the Center for Medicare and Medicaid Innovation. Health Aff. Published December 18, 2020. Accessed January 13, 2021. doi:10.1377/hblog20201216.672904 3. The Quality Payment Program. CMS website. October 25, 2016. Accessed January 13, 2021. https://www.cms.gov/newsroom/fact-sheets/ quality-payment-program

4. ASTRO responds to CMS Radiation Oncology Model: implementation delay and more reforms needed. News release. American Society for Radiation Oncology. September 18, 2020. Accessed January 13, 2021. https://www.astro.org/News-and-Publications/News-and-MediaCenter/News-Releases/2020/ASTRO-responds-to-CMS-Radiation-Oncology-Model-Imp Himmelstein DU, Lawless RM, Thorne D, Foohey P, Woolhandler S. Medical bankruptcy: still common despite the Affordable Care Act. Am J Public Health. 2019;109(3):431-433. doi:10.2105/AJPH.2018.304901
}

\section{Field Reports: Comments on Oncology Care Model Progress}

\author{
Basit Chaudhry, MD, PhD, founder \\ of Tuple Health
}

Feedback from the field is incredibly important when participating in new payment models like the Oncology Care Model, said Basit Chaudhry, MD, PhD, founder of Tuple Health.

With Oncology Care First being delayed at least a year now, where do you see opportunities for CMS to make some changes to the model based on the ongoing challenges practices have reported in the Oncology Care Model?

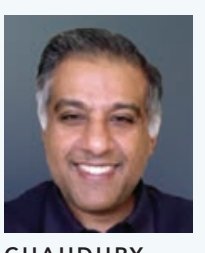

One of the things that Medicare talks about quite a bit in these payment models, particularly through CMMI [Center for Medicare and Medicaid Innovation], is that they're learning models. And I think that's the CHAUDHRY right spirit. You know, these kinds of changes are so complicated, even for an entity as large as Medicare, there's no way to get it right in the beginning. So, this whole tact of making things a learning approach, I think, is really critical. And I think it's very, very important to really think, from the payer perspective, really think carefully about what did work, what did not, and I know Medicare tries to do this, and then really implement those changes. And I think one of the big things that we've seen particularly for a model as complicated as OCM, is, I think, until you're actually living it and doing it directly, it's hard to know what it's like.

So, what we really hope is that feedback from the field is one of the drivers for change and OCF in the design. So, I think taking a human-centric or user-centered design approach towards the problem would be something l'd very much recommend and hope that we see payers do more and more.

\section{Amy Ellis, Chief Quality Officer,}

Northwest Medical Specialties

With so many different oncology alternative payment models (APMs) available, choosing the right ones to participate in might simply mean finding which ones already align with what a practice is doing, said Amy Ellis, chief quality officer at Northwest Medical Specialties.

Recently, the Community Oncology Alliance released a report that there are more than 30 oncology-related APMs. How does Northwest Medical Specialties determine which model to participate in?

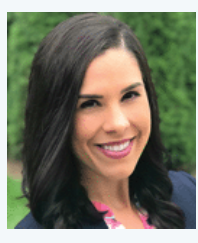

There's a lot of different APMs out there. And what we really try to do is look at the quality measures that that specific APM is requiring, or that specific payer, and how it aligns with our current programs. We don't want to get into a situation where we have 15 different programs with 15 different quality measure rules and methodologies, because there is a lot of administrative burden that takes place behind the scenes of all of these APMs, whether that be, you know, billing out the MEOS [Monthly Enhanced Oncology Services], or tracking the patients-all of these different things.

So, what we really try to do is find out what's aligned with what we're already doing. And of course, there's some wiggle room. If there's something that's trying to introduce a new measure for us, or maybe has a slightly different methodology, we're open to that. But when you get into a space where you have these completely different models, that means you may need more staff to support those models. I think those are the really the key things that we look at is really alignment between the models. 


\title{
Use of COTA Nodal Address Highlights Variance in Cancer Care Cost: An Interview With Donald M. Berwick, MD, MPP
}

\author{
MARY CAFFREY
}

CONTINUED FROM COVER

Now, new research has shown how much the cost of cancer care can vary within a multihospital system, something that must be avoided as health systems expand. In December, authors associated with the health care technology company COTA, Inc, published results that used the digital classification system the COTA Nodal Address (CNA), ${ }^{5}$ to reveal large differences in total cost of care for common cancers. ${ }^{6}$ Each CNA phenotype is developed through a peer-review process and includes evidence-based patient elements and disease-specific parameters.

For this study, authors used data from 4032 patients across the 16-hospital Hackensack Meridian Health system from January 2013 to January 2016. Costs were based on schedules from Medicare and Horizon Blue Cross Blue Shield of New Jersey-the state's largest insurer-and validated against other insurers. Cancer cases with the same CNA have the same clinical characteristics, and results showed that cases with the same CNA had the following ranges:

- $\quad$ Breast cancer care costs varied from $\$ 16,000$ to $\$ 88,000$

- Colorectal cancer care costs varied from $\$ 25,000$ to $\$ 222,000$

- $\quad$ Lung cancer care costs varied from $\$ 44,000$ to $\$ 424,000$.

Variation in cost among cancers with the same CNA was seen in both academic and community-based settings, and the use of clinical pathways did not eliminate variation. Results showed that the less common the cancer, the more variation increased.

Findings also show that the greatest opportunities for improvement were (1) making sure patients get on the right clinical pathway at the outset, based on comorbidities and genetic profile, and (2) eliminating unnecessary ancillary care.

Do the findings bolster the case for payer coverage of genetic or genomic testing? In an email, the paper's lead author, Andrew L. Pecora, MD, FACP, CPE, a founder of COTA and chief innovation officer, professor and vice president of cancer services at Hackensack Meridian Health and John Theurer Cancer Center, wrote: "We must know exactly what the patient is from a disease perspective. There can be no compromise on this, so genetic testing to assign the correct CNA is a must."

For insight into the results, Evidence-Based Oncology ${ }^{\mathrm{TM}}(E B O)$ spoke with the paper's senior author, Donald M. Berwick, MD, MPP, the former CMS administrator who is president emeritus and senior fellow at the Institute for Healthcare Improvement in Cambridge, Massachusetts. Berwick, a member of the faculty at Harvard Medical School, is well known for coining the term, the "triple aim," which refers to simultaneously improving the health of populations and the health care experience of patients while lowering the cost of care. ${ }^{7}$ The following has been edited for clarity:

EBO: Eliminating unnecessary variation in care is at the heart of what we talk about in value-based care. And yet, the argument is made that cancer care is different. Often, there's not a less expensive version of "the best" therapy. How do your findings on the use of the COTA Nodal Address speak to this issue?

BERWICK: I do think we want oncologists and other clinicians to be able to make adjustments in individualized patient care that they feel are needed. We need to trust the professional discipline and scientific integrity of the clinician. None of [these findings] should be converted into handcuffs or undue restrictions on clinicians. However, by studying variation, we can reveal variability in practice that they may not know about-and that can open the door to inquiry. We can imagine people saying: "Why do you do it that way? This is the way I do it." And we open exploration that's otherwise not possible, if we haven't turned the lights on to that variation.

Second, if there is justification for the variation based on underlying scientific evidence, and there are several different evidence-based pathways to care, but one of them consumes fewer resources or is easier to implement for patients and families and clinicians - all other things being equal-we should know that. If the claim is the outcomes are the same, then why don't we choose the approach that is less resource intense?

\section{"If there is justification for the variation based on underlying scientific evidence, and there are several different evidence- based pathways to care, but one of them consumes fewer resources or is easier to implement for patients and families and clinicians-all other things being equal- we should know that."}

\section{—Donald M. Berwick, MD, MPP, former CMS administrator, current president emeritus and senior fellow at the Institute for Healthcare} Improvement

Third, it's good to link the results, the resource use-which the COTA system allows-to outcomes. And although this is associational evidence, not experimental evidence, it still can be suggestive. If we begin to notice that certain channels or certain pathways of care seem to be associated with better outcomes for patients, we should notice that and use that to induce more disciplined experimental research. So, there's a lot of different ways that this can be put to use.

EBO: What accounts for so much differentiation in costs for the different pathways?

BERWICK: It's intensity of resource use. Basically, there are habits or patterns of utilization that do more testing or intervention, use a different assortment of medications, different frequency of visits. In general, we're looking at different intensity of patterns and resources based on clinical habits and local procedures.

We know from prior research [that] when I'm in a community, my practice tends to look like others in that community-even if it's very different from people in other communities, so we have these different local habits. Clinicians tend to stay local and learn from each other.

In oncology, that's less true; the oncology community is very organized together, using national and international data, but "

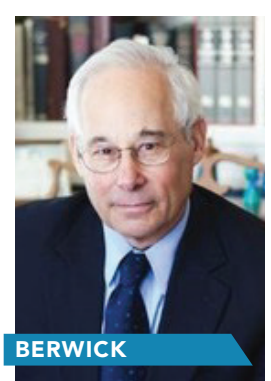

Donald M. Berwick, $M D, M P P$, former CMS administrator, current senior fellow at the Institute for Healthca Improvement 
these local work patterns and habits still really do matter. And a lot of the variation we're seeing reflect these local behaviors. Dr [John] Wennberg called it the surgical signature, which means it was the characteristic of the neighborhood. ${ }^{8}$

EBO: The findings show that there's less variation in cost in the more commonly seen cancers, such as breast or lung cancer, and more variation in the rare cancers-the rarer the cancer, the more variation we see. Is there a case to be made for greater data sharing in these rare cancers, so that we can close this gap?

BERWICK: Absolutely, yes. First, the phenomenon could be partly statistical, because the higher the sample size in a particular cell, just statistically, the less variation [we will see] among the entries in that cell. That's just a statistical fact. We would expect more variation in the lower sample size cells in the COTA system. That said, another important factor is scientific certainty: The less experience I have with the management of particular cancer, the more chance there is that variability and knowledge will begin to operate. And I'll be developing patterns that aren't based on large data sets. So yes, the ability to aggregate data for small or smaller incidents, and smaller prevalence, cancers or any disease, is a real asset. It's like what we discussed in the first question: It's a chance to raise some questions that otherwise couldn't be raised because we wouldn't have the data.

EBO: We often hear the about the need to balance too much variation with the need to treat the patient in front of me, kind of going back to the first question. What did the CNA findings say about the need to strike this balance?

BERWICK: Overall, the CNA findings suggest we are out of balance right now. Because I've been in this research field for so long, the amount of variation is not surprising, but it doesn't look ideal. I mean, this amount of variability in the care of cancers that are relatively common, for example, isn't scientifically easily defensible. So, number one, I think it does raise some questions as to whether the oncologic community and clinicians in general need to reconsider their commitments to simply using the best practice they can find.

Second, there is the need to adjust care to the local circumstance, to every individual patient. At the Institute for Healthcare Improvement, we always say, we need to practice medicine not only about what's the matter with you, but what matters to you.

Even if a person has the identical form of breast cancer or colon cancer, the individual's needs and preferences and what matters to the patient must be honored. That's going to be sensed locally, and we would expect that as clinicians adjust to individual needs, a very important and valuable form of variation will (and should) enter into the picture.

Also, although the COTA Nodal Address uses a lot of variables-and this is a data-intense way to explore patient variation (variation at the patient level)—it doesn't cover absolutely every variable that may be known. I suspect that some variation occurs in response to knowable characteristics about individual patients that don't happen to be variables in the in the coded data set. But knowing the size of the COTA data set and the number of things that are being that are being explored, [this] should raise questions for any clinicians who notices that they are outliers with respect to their peers.

EBO: So what are the next steps? Where are we and where do we go from here for closing the variation gaps?

BERWICK: I'd say 2 to 3 things. One is the power of the...COTA Nodal Address system. Any large registry grows with the size of the database, and so the aspiration would be that the amount of information in the database grows as fast as possible because the more data we have, the faster we can learn. So a growth of use is my hope.

A second would be partnership with the oncologic community-your readers. This should not be felt to be surveillance or enforcement or anything close to regulatory at this point. What's important is partnerships so that the curiosity and the scientific bias of the scientific intent of clinicians get raised. I hope that the COTA system can become better known to the oncologic community, so they can make use of it and ask the questions they probably want to be able to ask.

Third, there's probably a patient side of this-that patients deserve voice and control over their lives and care. There are, of course, the mathematical characteristics of the CNA that are a little complicated, but they're not insanely complicated. And I think patients and families could come to understand the COTA system as a way to illuminate variation in their own care and raise questions about, "Well, why do you do it that way, as opposed to this way?"
There may be very important uses for research. There's an obvious potential nexus between the research community and the COTA system, as COTA begins to reveal unexpected variability that should raise the curiosity of researchers, who could say, "We're doing this 4 different ways. Which is the best?" I think it could induce some serious research that would be to the benefit of patients.

On this cost side, the amount of variation observed does suggest that very likely a lot of money is being wasted. And it's not just money, it's time-of patients and families and clinicians. And it's also out-of-pocket costs, because a lot of the health care costs even in the insured population are coming out of patients' pockets. If it turns out that there's variation in resource use, but not in outcomes, that should give us an opportunity to actually look at ways of doing what we should do, which is to reduce health care costs without harming anyone-in fact, making things better. •

DISCLOSURE

Berwick has an equity interest in COTA, Inc.

\section{REFERENCES}

1. Diaz A, Paredes AZ, Hyer JM, Pawlik TM. Variation in value among hospitals performing complex cancer operations. Surgery. 2020;168(1):106-112 doi:10.1016/j.surg.2020.03.010

2. Ellis P, Sandy LG, Larson AJ, Stevens SL. Wide variation in episode costs within a commercially insured population highlights potential to improve the efficiency of care. Health Aff. 2012;31(9):2084-2093. doi: 10.1377/hlthaff.2012.0361

3. Oncology Care Model. CMS website. Updated September 14, 2020 Accessed January 10, 2021. https://innovation.cms.gov/innovation-models/oncology-care

4. Baumgardner J, Shahabi A, Zacker C, Lakdawalla D. Cost variation an savings opportunities in the Oncology Care Model. Am J Manag Care. 2018;24(12):618-623.

5. Pecora AL, Norden AD, Hervey J, et al. Development of a precise, clinically relevant, digital classification schema for cancer. JCO Clin Cancer Inform. 2018;2:2-10. doi:10.1200/CCI.18.00006

6. Pecora AL, Ip A, Wang CK, et al. The COTA Nodal Address model: a novel digital classification system identifies variances in cancer care cost to support value based care delivery. J Prec Med 2020;6(4): https:/ / www. thejournalofprecisionmedicine.com/the-cota-nodal-address-model-a-novel-digital-classification-system-identifies-variances-in-cancer-care-cost-to-support-value-based-care-delivery/ Berwick D, Nolan TW, Whittington J. The triple aim: care, health, cost. Health Aff. 2008; 27(3):759-769. doi:10.1377/hlthaff.27.3.759

8. Weinstein JN, Bronner KK, Morgan TS, Wennberg JE. Trends and geographic variations in major surgery for degenerative diseases of the hip, knee, and spine. Health Aff. 2004;23(suppl 2):VAR-81-VAR-89. doi:10.1377/hlthaff.var.81

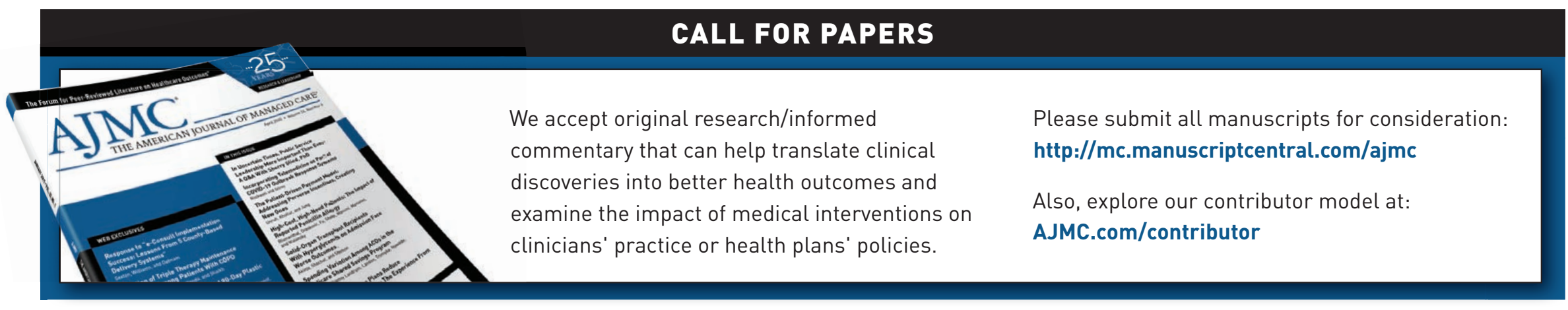




\title{
ACCC and ASCO Collaborative Targets Disparities in Cancer Clinical Trials
}

\author{
ROSE MCNULTY
}

\section{CONTINUED FROM COVER}

A recent report from the American Cancer Society highlighted persistently lower survival rates in Black cancer patients compared with White cancer patients in most cancer types, despite a steady overall decrease in cancer mortality in the United States from 1993 to 2018. ${ }^{3}$ Those findings further reinforce a lag in progress when it comes to closing long-known gaps in cancer outcomes.

"If clinical trials don't represent the individuals we treat, including those from racial and ethnic minority populations, the state of science suffers and patients with life-threatening conditions may miss out on the best-perhaps only-treatment option for their condition," 2020 ASCO president Lori J. Pierce, MD, FASTRO, FASCO, said in a statement when the collaboration was announced. "This initiative is of critical importance and we're honored to be working with ACCC to address longstanding barriers to diversity in cancer treatment trials."

Closing the gaps in cancer care will be no small task, but oncologists countrywide have embraced this cause, said Randall Oyer, $\mathrm{MD}$, medical director of the oncology program at Penn Medicine Lancaster General Health and 2020-2021 president of the ACCC. Oyer told Evidence-Based Oncology ${ }^{\mathrm{TM}}$ (EBO) that a request for ideas (RFI) released by the collaborating entities over the summer received a robust response from member practices nationwide, "highlighting how important this issue is to investigators across the country and how people have already started to work on the problem."

For insight and updates on the collaboration, EBO spoke with Oyer about the initiative and the disparities it aims to overcome. The following has been edited lightly for clarity:

EBO: First, can you discuss the need for greater diversity in clinical trial populations?

OYER: To begin, it's a matter of equity for all patients to be able to benefit from the advances that we have through cancer research. Secondly, applying trial results to people who would not have been eligible for a clinical trial has been associated with safety issues, and applying trial results to people who aren't invited to participate limits the generalization of the results and what we learn from the trial.

EBO: What went into the decision to collaborate with ASCO on the initiative to increase minority participation in clinical trials?

OYER: Both ASCO and ACCC have a very strong mission to apply results across all communities and have a very strong social mission. And truthfully, the CEO of ASCO, Dr Cliff Hudis, noticed the similarity between this year's presidential theme in ASCO where Dr Lori Pierce had chosen social determinants of health, and the presidential theme for ACCC, which was moving clinical trials out into the community. Clearly, there was overlap, and focusing on Blacks and other ethnic minorities who have been traditionally underrepresented in cancer treatment trials seemed to be something that we could do better together.

EBO: The window for idea submission closed in late August. How was the response rate?

OYER: About 30 thoughtful responses came in from ASCO and ACCC members, really highlighting how important this issue is to investigators across the country and how people have already started to work on the problem. So we were really pleased with the response, and there were some top-notch ideas.

EBO: What are some of the potential barriers to clinical trial participation for minority populations that you think might be the simplest to resolve in terms of strategy and resources?

OYER: There are many barriers to clinical trial participation for Blacks and [other] minorities, and actually, none is simple. But the simplest first step would be to offer trials to people. We've learned through our investigation process that we need to change the entire clinical trials ecosystem. There are complex issues having to deal with building trust in individuals and communities; providing trials for patients that match the conditions they have and for which they're eligible; recognizing bias in providers and systems, and taking steps to educate and mitigate against that; and addressing practical issues that impede a person's opportunity to participate in a clinical trial, such as transportation or time off from work, for example.

EBO: The RFI noted that a recent review found that there was a decrease in minority participation in clinical trials from 2003 to 2016. Why do you think those numbers appear to be shrinking despite increased focus on health care disparities in that time frame, and in recent years?

OYER: Well, it depends upon the type of cancer. In this study of National Cancer Institute trials, Blacks had lower enrollment fractions in breast, colorectal, and lung cancer trials, but not in prostate cancer trials. So it really means that we have to look at what the differences might have been in terms of study design, study marketing, and recruitment strategies. But the investigators didn't comment on that, so we can't really speculate.

EBO: Are there any specific strategies to address long-standing barriers to clinical participation? How does the new ACCC and ASCO collaborative aim to avoid similar results?

OYER: Well, I don't think we have a knowledge gap. I think we're seeing a dissemination gap, and I think we're seeing an investment gap. The problem has been documented: Blacks and other ethnic minorities are enrolled to a lower extent than others on cancer treatment trials, and our investigators, a review of the landscape, and our steering group have advised that we need an entire ecosystem to address this with solutions at the patient level, the system level, at the community level, at the provider level, and even in trial design.

\section{REFERENCES}

1. Loree JM, Anand S, Dasari A, et al. Disparity of race reporting and representation in clinica trials leading to cancer drug approvals from 2008 to 2018. JAMA Oncol. 2019;5(10):e191870. doi:10.1001/jamaoncol.2019.1870

2. Duma N, Aguilera JV, Paludo J, et al. Representation of minorities and women in oncology clinical trials: review of the past 14 years. J Oncol Pract. 2018;14(1):el-e10. doi:10.1200/ JOP.2017.025288

Siegel RL, Miller KD, Fuchs HE, Jemal A. Cancer Statistics, 2021. CA Cancer IClin. Published online January 12, 2020. doi:10.3322/caac.21654

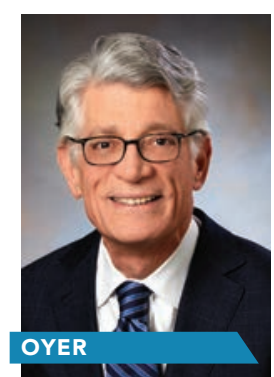

Randall Oyer, MD, 20202021 ACCC president medical director of the oncology program at Penn Medicine Lancaster General Health

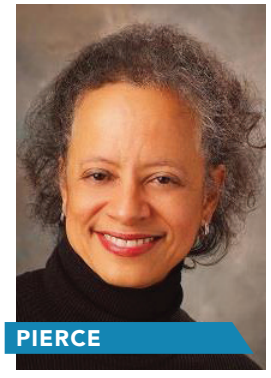

Lori J. Pierce, $M D$, FASTRO, FASCO, professor, and Vice Provost for Academic and University of Michigan, University of Michigan,

\section{cure}

Why It's Never Too Soon to Consider Clinical Trials as a Treatment Option http://bit.ly/3rOkRnB 


\section{DARZALEX Faspro'm}

(daratumumab and hyaluronidase-fihj)

Injection for subcutaneous use |1,800 mg/30,000units

\section{Permanent J-Code Now Available}

\section{J9144}

injection, daratumumab,

$10 \mathrm{mg}$ and hyaluronidase-fihj

The permanent $\mathrm{J}$-code is exclusive to DARZALEX FASPRO'TM and replaces the miscellaneous Healthcare Common Procedure Coding System (HCPCS) codes used across several sites of care.

Please be sure your systems are updated for claims with dates of service on, or after, January 1, 2021.

janssen CarePath
Call a Janssen CarePath Care Coordinator at 877-CarePath (877-227-3728), Monday-Friday, 8:00 AM to 8:00 PM ET.

For more information on the J-code for DARZALEX FASPROTM, please visit JanssenCarePath.com.

This document is presented for informational purposes only and is not intended to provide reimbursement or legal advice, nor does it promise or guarantee coverage, levels of reimbursement, payment, or charge. Similarly, all HCPCS codes are supplied for informational purposes only and represent no statement, promise, or guarantee by Janssen Biotech, Inc., that these codes will be appropriate or that reimbursement will be made. It is not intended to increase or maximize reimbursement by any payer. Laws, regulations, and policies concerning reimbursement are complex and are updated frequently. While we have made an effort to be current as of the issue date of this document, the information may not be as current or comprehensive when you view it. We strongly recommend you consult the payer organization for its reimbursement policies. 